\title{
Ubèr ein Bimetallproblem in der Ebene
}

\author{
L. JENTSOH
}

Es werden Existenz- und Eindeutigkeitssätze für Rand-Kontakt-Aufgaben der ebenen Ėlastostatik und Thermoclastostatik für stückweise homogene Scheiben, deren elastisch homogenen Teile längs einer Geraden zusammengeklebt sind, in einer geeigneten Klasse regulärer Vek: toren bewiesen. Das Problem wird mit Hilfe des.Kontakttensors der Elastostatik für zwei fest verbundene. Halbebenen auf ein Integralgleichungssystem zurückgeführt, das neben singulären Integralen singuläre Integrale mit feststehenden Singularitäten enthält. Aus der Theorie von Dodochava folgt, daB der Integraloperator im Raum $L_{2}(S)$ (S Rand der Scheibe) oin Noetheroperator mit Index Null ist.

Рассматриваются пл̀оские гранично-контактные задачи әластостатики и термозластостатики для кусочно-однородных сред, однородные части которых склеиваются вдоль прлмой. Доказываются теоремы существования и единственности решения в соответствующем пространстве регулярных векторов. Испольауя контактный тензор при рассмотрении эластостатики двух склеенных полуплоскостей сводим задачу к системе сингулярных интегральных урапнений с пеподвижными особенностями. Нетеровость сингулнрного оператора в пространстве $L_{2}(S)$ (S-граница плоской области) доказана с помощью теории Дудучавы.

Existence'and uniqueness theorems for boundary-contact-problems of plane elastostatics and thermoelastostatics are given in a proper class of regularity, when the thermoelastic constants. are piecewise constant and discontinuous along a straight line. The problem is reduced with the aid of the contact-tensor of clastostatics for two composite half-planes to a system of integral equations with fixed singularities. From the theory of Duducrivis it follows, that the integral operator is Noetherian in the space $L_{2}(S)$ ( $S$ boundary of the disc) with index zero.

.Betrachtet wird die zweite Randkontaktaufgabe der Thermoelastostatik in 'der Ebene, wenn die geradlinige Trennlinie zwischen den beiden elastisch homogenen Teilen bis zum äußeren Rand reicht. Der Fall, daß die Einschlüsse aus anderem Material ganz im Innern liegen, ist sowohl in der Ebene (s. [21, 18]) als im Raum (s. [16; Kap. XII], [5]) behandelt worden. Wir wählen für unser Problem die Kontaktbedingung, die der festen Verbindung der homogenen Teile entsprichit. Andere Kontaktbedingungen sind Gegenstand der Arbeiten $[1,6-11,14,15,17,19]$. Die in [12] im räumlichen Fall dargelegte Idee, das Problem mit Hilfe des Kontakttensors der Elastostatik für zwei aneinandergrenzende Halbräume auf ein Integralgleichungssystem über den Rand des Gesamtgebietes zurückzuführen, wird in dieser Arbeit im ebenen Fall genau dargelegt. Sitzt die Randkurve auf der geradlinigen Trennlinic senkrecht auf, erhält .man ein singuläres Integralgleichungssystem mit feststehenden Singularitäten, auf das die Theorie von Duduchava [3] anwendbar ist. Es wird ein Existenz- und Eindeutigkeitssatz in einer geeigneten Klasse regulärer Vektoren bewiesen. Aussagen iiber das Singularitätsverhalten in der Ecke eines aus verschiedenen isotropen homogenen Keilen zusammengesetzten Körpers sind in $[25,2]$ enthalten. 


\section{Formulierung des Problems}

In einem kartesischen $x_{1} x_{2}$-Koordinatensystem mit den Basisvektoren $\boldsymbol{i}_{1}, \boldsymbol{i}_{2}$ betrachten wir das Gebiet $D$ mit dem Rand $S$, das die Lage des elastischen Materials bestimmt. Das Material sei stückweise homogen, die Laméschen Moduln und den linearen Wärmeausdehnungskoeffizienten bezeichnen wir in $D^{+}=D$ $n\left\{x=\left(x_{1}, x_{2}\right)^{T}: x_{2}>0\right\}$ mit $\lambda_{1}, \mu_{1}, \alpha_{1}$. und in $D^{-}=D \cap\left\{x: x_{2}<0\right\}$ mit $\lambda_{0}, \mu_{0}, \alpha_{0}$. Es sei $S_{0}=D \cap\left\{x: x_{2}=0\right\}$ ein Intervall (s. Abb: 1) und $S^{+}=S \cap\left\{x: x_{2}>0\right\}$, $S^{-}=S \cap\left\{x: x_{2}<0\right\}$. 'Die Normaleneinheitsvektoren' $n$ seien wie in Abb. 1 orientiert. Vektoren fassen wir stets als Spaltenmatrizen auf, für das Skalarprodukt $\boldsymbol{a}^{T} \boldsymbol{b}$ schreiben wir auch $\boldsymbol{a} \cdot \boldsymbol{b}$. :

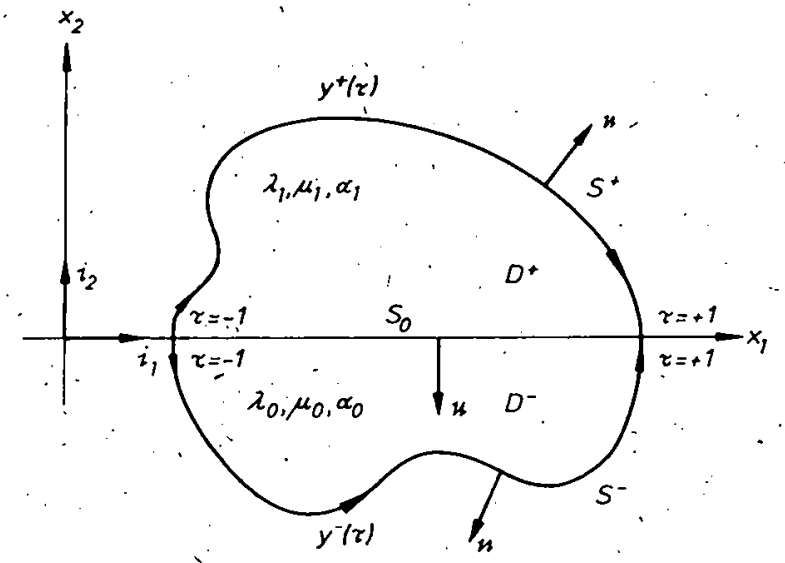

Abb. 1

Definition 1.1: Es sei $S \subset C_{n}^{1, \alpha}(0<\alpha \leqq 1)$, wenn $S \subset C^{1, \alpha}$ und $S$ die Gerade $x_{2}=0$ in genau zwei Punkten senkrecht schneidet (vgl. [18, Def. 2.1])

Falls $S \subset C_{n}^{1, \alpha}$, dann besitzt $\overline{S^{ \pm}}$eine Parameterdarstellung ,

$$
y^{ \pm}(\tau)=y_{1}^{ \pm}(\tau) i_{1}+y_{2}^{ \pm}(\tau) i_{2}, \quad-1 \leqq \tau \leqq+1
$$

mit $\quad \dot{y}_{1}^{ \pm}( \pm 1)=0, \quad \dot{y}_{2}^{ \pm}(1)=\mp 1, \quad \dot{y}_{2}^{ \pm}(-1)= \pm 1, \quad y^{+}( \pm 1)=y^{-1}( \pm 1) \quad$ und $y_{j}^{ \pm}(\tau)$ $\in C^{1, a}([-1,+1]),\left|\dot{y}^{ \pm}(\tau)\right| \neq 0$ für $-1 \leqq \tau \leqq+1$.

Einige Bezeichnungen: $K\left(x_{0}, r\right)=\left\{x:\left|x-x_{0}\right|<r\right\}, \quad K_{\varepsilon}=K\left(y^{+}(-1), \varepsilon\right)$ $\cup K\left(y^{+}(+1), \varepsilon\right), D_{\varepsilon}=D \backslash K_{\varepsilon}, D_{\varepsilon}^{ \pm}=D^{ \pm} \backslash K_{\varepsilon}$, für $x \in K_{\varepsilon_{0}}\left(\varepsilon_{0}>0\right.$ genügend klein $)$ sei $\varrho_{x}$ der kürzeste. Abstand des Punktes $x$. von $S$ auf der Parallelen zu $x_{2}=0$, . $C D=\{x: x \notin \bar{D}\}, \quad C D^{+}=\left\{x: x \notin \overline{D^{+}}, x_{2}>0\right\}, \quad C D^{-}=\left\{x: x \notin \overline{D^{-}}, x_{2}<0\right\}, \quad C D_{\varepsilon}$ $=C D \backslash K_{\varepsilon}, C D_{\varepsilon}^{ \pm}=C D^{ \pm} \backslash K_{\varepsilon}$.

Definition 1.2: Ein Vektor $\boldsymbol{u}=\boldsymbol{u}(x)=u_{1}(x) i_{1}+u_{2}(x) \boldsymbol{i}_{2}$ heiße in $D$ regulär, wenn

$$
\begin{aligned}
& \left.\boldsymbol{u}\right|_{D^{+}} \in C^{0}\left(\overline{D_{\varepsilon}^{+}}\right) \cap C^{1}\left(\overline{D_{\varepsilon}^{+}}\right) \cap C^{2}\left(D^{+}\right), \\
& \left.\boldsymbol{u}\right|_{D^{-}} \in C^{0}\left(\overline{D_{\varepsilon}^{-}}\right) \cap C^{1}\left(\overline{D_{\varepsilon}}\right) \cap C^{2}\left(D_{-}^{-}\right) \text {für jedes } 0<\varepsilon<\varepsilon_{0},
\end{aligned}
$$

$u$ beschränkt in $D^{+} \cup D^{-}$und $\frac{\partial u}{\partial x_{j}}=\mathcal{O}\left(\frac{1}{\sqrt{\varrho_{x}}}\right)$ für $x \in\left(D^{+} \cup D^{-}\right) \cap K_{\varepsilon_{0}}$. 
Es heiße $u$ in $C D$, regulär, wenn

$$
\begin{aligned}
& \left.\boldsymbol{u}\right|_{C D^{+}} \in C^{0}\left(\overline{C D_{\varepsilon}^{+}}\right) \cap C^{1}\left(\overline{C D_{\varepsilon}^{+}}\right) \cap C^{2}\left(C D^{+}\right), \\
& \left.\boldsymbol{u}\right|_{C D^{-}} \in C^{0}\left(\overline{C D_{\varepsilon}^{-}}\right) \cap C^{1}\left(\overline{C D_{\varepsilon}^{-}}\right) \cap C^{2}\left(C D^{-}\right) \text {fiir } 0<\varepsilon<\varepsilon_{0},
\end{aligned}
$$

$u$ beschränkt in $C D^{+} . \cup C D^{-}$und $\frac{\partial u^{\circ}}{\partial x_{j}}=\mathcal{O}\left(\frac{1}{\sqrt{\varrho_{x}}}\right)$ für $x \in\left(C D^{+} \cup C D^{-}\right) \cap K_{c_{0}}, \frac{\partial u}{\partial x_{j}}$
$=\mathcal{O}\left(|x|^{-2}\right)$ für $|x|>R_{0}$.

Definition 1.3: Ein Vektor $\boldsymbol{u}$ ist Lösung des Problems $\mathrm{I}(D ; \boldsymbol{F}, \theta ; \boldsymbol{w} ; \hat{\boldsymbol{w}}, \boldsymbol{P})$ bzw. des Problems II $(D ; \boldsymbol{F}, 0 ; \boldsymbol{P} ; \hat{\boldsymbol{w}}, \hat{\boldsymbol{P}})$, wenn $\boldsymbol{u}$ in $D$ regulär ist und

a) der Differentialgleichung

$$
\begin{aligned}
& A u=\left(2 \mu_{1}+3 \lambda_{1}\right) \alpha_{1} \operatorname{grad} \theta-F^{\prime} \text { in } D^{+}, \\
& \underset{(1)}{A u} \doteq\left(2 \mu_{0}+3 \lambda_{0}\right) \alpha_{0} \operatorname{grad} \theta-F \text { in } D^{-},
\end{aligned}
$$

b) der Randbedingung $\boldsymbol{u}=\boldsymbol{w}$ auf $S$ bzw.

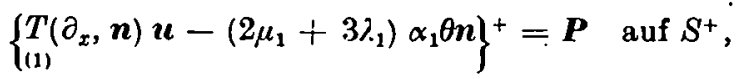

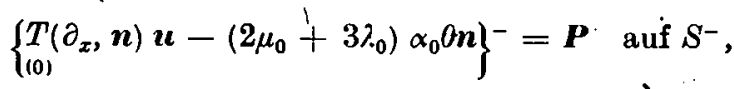

c) der Kontaktbedingung

$$
\begin{aligned}
& \{\boldsymbol{u}\}^{+}-\{\boldsymbol{u}\}^{-}=\hat{\boldsymbol{w}} \text { auf } S_{\mathbf{0}},
\end{aligned}
$$

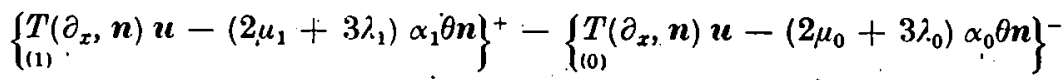

$$
\begin{aligned}
& =\hat{\boldsymbol{P}} \text { auf } S_{0} \text {. }
\end{aligned}
$$

genügt.

Es ist $\boldsymbol{u}$ Lösung des Problems I $(C D ; \boldsymbol{F}, \theta ; \boldsymbol{v} ; \hat{\boldsymbol{w}}, \hat{\boldsymbol{P}})$ bzw. des Problems $\mathrm{II}(C D ; \boldsymbol{F}, \boldsymbol{0}$; $\boldsymbol{P} ; \hat{\boldsymbol{v}}, \hat{\boldsymbol{P}})$, wenn $\boldsymbol{u}$ in $C D$ regulär ist, $(1.1+)$ in $C D^{+},(1.1-)$ in $C D^{-}, \boldsymbol{u}=\boldsymbol{w}$ auf $S$ bzw. (1.2) und (1.3), (1.4) auf $\left\{x: x_{2}=0\right\} \backslash \bar{S}_{0}$ gilt. Hierbei ist

$$
\begin{aligned}
& A \boldsymbol{u}=\mu_{i} \Delta \tilde{\boldsymbol{x}}+\left(\dot{\lambda}_{i}+\mu_{i}\right) \text { graddiv } \boldsymbol{u}, \\
& \text { (I) }
\end{aligned}
$$

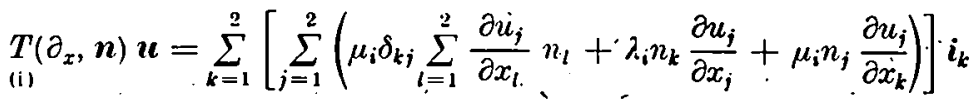

und $\{\cdot\}^{+},\{\cdot\}^{-}$bezeichnet den Grenzwert gegen einen Randpunkt von $D^{+}, D^{-}$auf der Normalen.

Bemerkung: Das Problem I $(D ; F, 0 ; 20 ; 0,0)$ bzw. II $(D ; \boldsymbol{F}, 0 ; \boldsymbol{P} ; 0,0)$ kann aufgefaßt werden als quasistatisches Wärmespannungsproblem zur Ermittlung des Vérschiebungsvektors $u^{\prime}$ in einem unendlich langen Zylinder mit dem Querschnitt $D$ im ebenen Deformations. zustand oder in einer Scheibe, wenn die elastisch homogenen Teile längs $S_{0}$ fest verbunden sind und am Rand der Verschiebungsvektor $w$ bzw. die Normalspannung $P$ vorgegeben sind und im Innern des Körpers ein äußeres Kraftfeld $\boldsymbol{F}$ und Temperaturfeld $\theta$ wirken. Dabei kann $\theta$ beliebig vorgegeben sein, Lösung einer Randkontaktaufgabe für die stationäre Wärmeleitungsgleichung oder Lösung eines Anfangs-Randkontaktwertproblems für die instationäre Wärmeleitungsgleichung zu einem festen Zeitpunkt sein. 
Eindeutigkeitssatz: Die Probleme $\mathrm{I}(D ; 0,0 ; 0 ; 0,0)$ und $\mathrm{I}(C D ; 0,0 ; 0 ; 0,0)$ haben nur die triviale Lösung, das Problem $\mathrm{II}(D ; 0,0 ; 0 ; 0,0)$ hat genau $\mathscr{L}\left\{\boldsymbol{a}_{1}, \boldsymbol{a}_{2}, \boldsymbol{a}_{3}\right\}$ und das Problem II $(C D ; 0,0 ; 0 ; 0,0)$ hat genau $\mathscr{L}\left\{a_{1}, a_{2}\right\}$ als Lösung.

Beweis: Die Vektoren $a_{1}=i_{1}, a_{2}=i_{2}, a_{3}=-x_{2} i_{1}+x_{1} i_{2}$ und damit der von $a_{1}, a_{2}, a_{3}$ aufgespannte lineare Raum $\mathscr{L}\left\{a_{1}, a_{2}, a_{3}\right\}$ sind offenbar Lösung des Pro- : blems II $(D ; 0,0 ; 0 ; 0,0)$. Ebenso ist $\mathscr{L}\left\{a_{1}, a_{2}\right\}$ Lösung des Próblems II $(C D ; \mathbf{0}, 0 ;$ $0 ; 0,0)$.

Śei nun $\boldsymbol{u}$ Lösung des Problems $\mathrm{I}(D ; \mathbf{0}, 0 ; \mathbf{0} ; \mathbf{0 , 0})$ oder $\operatorname{II}(D ; 0,0 ; 0 ; 0,0)$. Wenden wir den Integralsatz (3.11) in [18] an auf $D^{+} \backslash Q_{\varepsilon}, D^{-} \backslash Q_{\varepsilon}$ mit $Q_{\varepsilon}=\{x \in D$ : $\left(\left|x_{2}\right|<\varepsilon, \varrho_{x}<\varepsilon\right\}$, dann folgt für die elastische Energie (s. [18: (3.14)])

$$
E(\boldsymbol{u}(x))=\lambda\left(u_{1,1}+u_{2,2}\right)^{2}+2 \mu\left(u_{1,1}^{2}+u_{2,2}^{2}\right)+\mu\left(u_{1,2}+u_{2,1}\right)^{2}
$$

$\lambda=\lambda_{1}, \mu=\mu_{1}$ für $x_{2}>0, \lambda=\lambda_{0}, \mu=\mu_{0}$ für $\dot{x_{2}}<0$ )

$$
\int_{D \backslash Q_{\varepsilon}} E(\boldsymbol{u}(x)) d x=\int_{\partial Q_{e} \backslash s}^{\prime} \boldsymbol{u} \cdot T\left(\partial_{x}, \boldsymbol{n}\right) \boldsymbol{u}(x) d_{x^{s}}=\mathcal{O}(\sqrt{\varepsilon}) .
$$

Also ist $\int_{D} E(\boldsymbol{u}(x)) d x=0$. Da $\boldsymbol{u}$ homogene Kontaktbedingungen erfüllt, folgt hieraus . (s. [18: Hilfssatz: 3.1]) $u \in \mathscr{L}\left\{a_{1}, a_{2}^{\prime}, a_{3}\right\}$. Analog schließt man bei den Außengebietaufgaben. Dort scheidet $\boldsymbol{a}_{3}$ wegen des Verhaltens im Unendlichen aus. Bei der ersten Randbedingung kommt nur $\boldsymbol{u} \equiv \mathbf{0}$ in Frage

Notwendige Lösbarkeitsbedingung: Hat das $\operatorname{Problem~} \operatorname{Ir}(D ; \boldsymbol{F}, \theta ; \boldsymbol{P} ; \hat{\boldsymbol{w}}, \hat{\boldsymbol{P}})$ eine Lösung, dann gilt

$$
\int_{D} \dot{F} \cdot \boldsymbol{a}_{k} d x+\int_{s} \boldsymbol{P} \cdot \boldsymbol{a}_{k} d s+\int_{s_{0}} \boldsymbol{P} \cdot \boldsymbol{a}_{k} d s=0, \quad k=1,2,3 .
$$

Dies folgt durch Anwendung des Integralsatzes (3.13) in [18] auf $D^{+} \backslash Q_{\varepsilon}, D^{-} \backslash Q_{\varepsilon}$ und anschließenden Grenziibergang $\varepsilon \rightarrow+0$

\section{Potentialtheorie}

Die Lösung des Problems II $(D ; \mathbf{0}, 0 ; \boldsymbol{P} ; \mathbf{0}, \mathbf{0})$ suchen wir in Form des Potentials

$$
\boldsymbol{V}(x, \varphi):=\frac{1}{\pi} \int_{s} \mathbf{G}(x, y) \boldsymbol{\varphi}(y) d_{y} s .
$$

Dabei ist $\mathbf{G}(x, y)$ der Greensche Kontaktiensor der Elastostatik für zwei fest verbundene Halbebenen mit unterschiedlichen Laméschen Moduln, der in [13] explizit berechnet wurde.

Satz 2.1: Es sei $\left.S \subset C_{n}{ }^{1, \alpha}, \varphi \in L_{2}{ }^{2}(S) \cap C^{0, \beta}\left(S \backslash K_{\varepsilon}\right)^{1}\right)$ für jedes feste $\varepsilon>0$

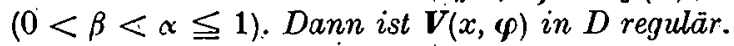

Beweis: Die Differenzierbarkeitseigenschaften von $V(x, \varphi)$ folgen aus bekannten Eigenschaften des elastischen Potentials der einfachen Schicht (s. $[21,18,22]$; vgl. auch [16: S. 226]). Wir brauchen nun nur noch die Abschätzungen in $K_{\varepsilon_{0}} z u$ zeigen. Dazu wird nur die Voraussetzung $\varphi \in L_{2}^{2}(S)$ benötigt. Es genügt, die Abschätzung in einer Umgebung von $y_{0}=y^{+}(+1)$ zu zeigen. Wir wählen $\delta>0$ so,

1) Es ist $L_{2}^{k}(M)=\left\{\varphi=\left(\varphi_{1}, \ldots, \varphi_{k}\right)^{T}: \varphi_{j} \in L_{2}(M)\right\}$ und $\|\varphi\|^{2}=\int_{M} \sum_{j=1}^{k}\left|\varphi_{j}\right|^{2} d M$. 
$\operatorname{da} ß$ für $x \in K\left(y_{0}, \delta\right), y \in S \cap K\left(y_{0}, \delta\right)$ gilt

$$
\frac{\left|x_{2}-y_{2}\right|}{\left|\varrho_{x}-\right| x_{1}-y_{i}||} \geqq 6 \text {. }
$$

Dann folgt leicht

$$
\frac{1}{2}\left[e_{x}^{2}+\left(x_{2}-y_{2}\right)^{2}\right] \leqq|x-y|^{2} \leqq \frac{3}{2}\left[\varrho_{x}^{2}+\left(x^{2}-y_{2}\right)^{2}\right] .
$$

Für $|x-y| \geqq \frac{\delta}{2}$ ist $\mathbf{G}(x, y)$ und $\frac{\partial \mathrm{G}(x, y)}{\partial x_{j}}$ beschränkt und für $|x-y| \leqq \delta$ gilt $\mathbf{G}(x, y)=\mathscr{O}\left(\ln \frac{1}{|x-y|}\right), \frac{\partial \mathbf{G}(x, y)}{\partial x_{i}}=\mathcal{O}\left(\frac{1}{|x-y|}\right)$. Seinuin $\dot{x} \in K\left(y_{0}, \frac{\delta}{2}\right) \cap\left(D^{+} \cup D^{-}\right)$, dann folgt unter. Verwendung der Schwarzschen Ungleichung

$$
\begin{aligned}
\left|V_{k}(x, \varphi)\right| & \leqq C_{1} \int_{s \backslash K\left(y_{0}, \delta\right)} \sum_{j=1}^{2}\left|\varphi_{j}(y)\right| d_{y} s+C_{2} \cdot \int_{s \cap} \ln \frac{1}{|x-y|} \cdot \sum_{j=1}^{2}\left|y_{j}(y)\right| d_{y} s \\
& \left.\leqq C_{3}\|\varphi\|+2 C_{2}\right] \sqrt{\int_{s \cap}\left(\ln \frac{1}{|x-y|}\right)^{2} d_{y} s\|\varphi\|_{0}}
\end{aligned}
$$

Unter Beachtung von (2.2) kann man zeigen, daß das Integral unter der Wúrzel

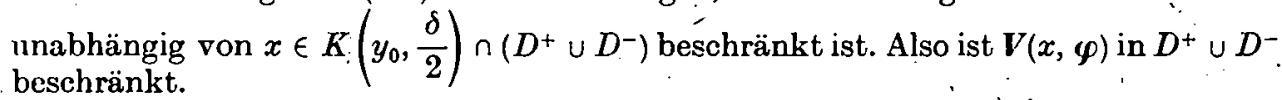

Analog ist

$$
\left|\frac{\partial V_{k}(x, \varphi)}{\partial x_{j}}\right| \leqq C_{4}\|\varphi\|+C_{5} \sqrt{\int_{s \cap K\left(\nu_{0}, \delta\right)} \frac{1}{|x-y|^{2}} d_{y} s}\|\varphi\| .
$$

Nun ist wegen (2.2)

$$
\begin{aligned}
\int_{s \cap K\left(y_{0}, \delta\right)} \frac{1}{|x-y|^{2}} d_{y^{s}} & \leqq 2 \cdot \int_{s \cap K\left(y_{0}, \delta\right)} \frac{1}{\varrho_{x}^{2}+\left(\dot{x}_{2}-y_{2}\right)^{2}} d_{y} s \\
& \leqq C_{6} \int_{t_{1}}^{t_{1}} \frac{1}{\varrho_{x}^{2}+\left(x_{2}-t\right)^{2}} d t=\left.C_{6} \frac{1}{\varrho_{x}} \arctan \frac{u}{\varrho_{x}}\right|_{u=x_{2}-t_{1}} ^{u=x_{2}-t_{1}} \\
\therefore \quad & \leqq C_{0} \pi \frac{1}{\varrho_{x}}
\end{aligned}
$$

Also ist $\frac{\partial \boldsymbol{V}(x, \varphi)}{\partial x_{i}}=\mathcal{O},\left(\frac{1}{\sqrt{\varrho_{x}}}\right)$

Utber dâs Verhalten von $\boldsymbol{V}(x, \varphi)$ im Unendlichen gibt der folgende Satz Auskunft. Satz 2.2: Sei $\varphi \in L_{1}^{\prime 2}(S)$. Fürr die Gültigkeit jeder der beiden Abschätzungen $\mathbf{V}(\dot{x}, \varphi)$ $=o(1)$ und $\frac{\partial \boldsymbol{V}(x, \boldsymbol{\rho})}{\partial x_{j}}=\mathcal{O}\left(|x|^{-2}\right)$ ist $\int_{S_{1}} \boldsymbol{\varphi}(y) d_{y} s=\mathbf{0}$ notwendig und hinreichend. 
Dieser Satz wird im Prinzip wie der entsprechende Satz für das klassische Potential der einfachen Schicht bewiesen (s. [23: S. 234]), nur sind hier die Rechnungen aufwendigér. Man muß bein Beweis die Eigenschaften von $\mathrm{G}(x, y)$ ausnutzen.

Aus Satz 2.1 und Satz 2.2 folgt eine weitere Aussage.

Satz. 2.3: $E s$ sei $S \subset C_{n}^{1, \alpha}, \varphi \in L_{2}{ }^{2}(S) \cap C^{0, \beta}\left(S \backslash K_{\varepsilon}\right)$ für jedes feste $\varepsilon>0$ $(0<\beta<\alpha \leqq 1)$ und $\int_{S} \varphi(y), d_{\nu} s=0$. Dann ist $\boldsymbol{V}(x, \varphi)$ in $C D$ regulár.

Auf Grund der Eigenschaften von $\mathrm{G}(x, y)$ (s. [13]) folgt aus Satz 2.1 noch eine Aussage.

Satz 2.4: Es. sei $S \subset C_{n}^{1, \alpha}, \varphi \in L_{2}{ }^{2}(S) \cap C^{0, \beta}\left(S \backslash K_{\varepsilon}\right)$ für jedes feste $\varepsilon>0$, $(0<\beta<\alpha \leqq 1)$. Dann ist $\mathrm{V}(x, \varphi)$ reguläre Lösung des Próblems $\mathrm{I}\left(D ; 0,0 ;\left\{\boldsymbol{V}\left(x_{0}, \boldsymbol{\rho}\right)\right\}\right.$; $\mathbf{0}, \mathbf{0})$.

Weiter benötigen wir den folgenden Satz.

Satz 2.5: Es sei $S \subset C_{n}^{1, \alpha},\left.\boldsymbol{u}_{p}\right|_{D^{+}}=\boldsymbol{u}_{p}{ }^{+} \in C^{1, \beta}\left(D^{+}\right) \cap C^{2}\left(D^{+}\right),\left.\boldsymbol{u}_{p}\right|_{D^{-}}=\boldsymbol{u}_{p}{ }^{-} \in C^{1, \beta}\left(D^{-}\right)$ $\cap C^{2}\left(D^{-}\right)(0<\beta<\alpha \leqq 1)$ und $\boldsymbol{u}_{p}{ }^{+}\left(y^{+}( \pm 1)\right)=\boldsymbol{u}_{p}{ }^{-}\left(y^{+}( \pm 1)\right)$. Dann ist

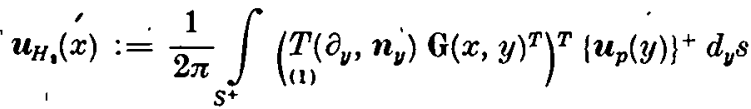

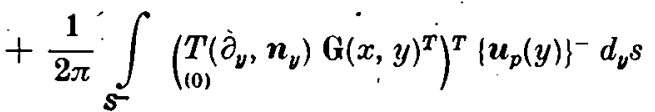

reguläre Lösung des Problems $\mathrm{I}\left(D ; 0,0 ;\left\{\boldsymbol{u}_{H_{\mathbf{2}}}\right\} ; 0,0\right)$ und für den Grenzwert

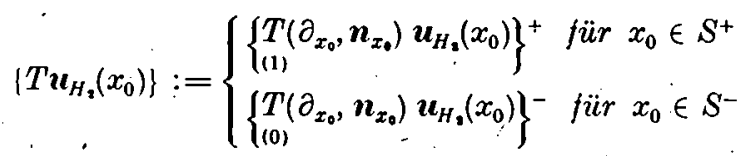

gilt $\left\{T u_{H_{\mathrm{a}}}\left(x_{0}\right)\right\} \in L_{2}^{2}(S) \cap C^{0, \beta}\left(S \backslash K_{\varepsilon}\right)$.

Beweis: Das Erfülltseiń der Differentialgleichung und der homogenen Kontaktbedingungen folgt aus den Eigenschaften von $\mathrm{G}(x, y)$, die Differenzierbarkeitseigenschaften folgen aus bekannten Ergebnissen über das elastische Potential der doppelten Schicht (vgl. [16: S. 226]). Nun zur Abschätzung in $K_{\iota_{\bullet}}$.

Aus der Bettischen Formel folgt (vgl. [18: (3.16)])

$$
\begin{gathered}
u_{p}(x)=-\frac{1}{2 \pi} \int_{s^{+}}\left(\int_{(1)}^{T}\left(\partial_{y}, n_{y}\right) \dot{G}(x, y)^{T}\right)^{T} u_{p}(x) d_{y} s \\
\quad-\frac{1}{2 \pi} \int_{s^{-}}\left(\begin{array}{l}
\left.T\left(\partial_{y}, n_{y}\right) \dot{G}(x, y)^{T}\right)^{T} u_{p}(x) d_{y} s
\end{array}\right.
\end{gathered}
$$

für $x \in D^{+} \cup D^{-}$. Damit ist

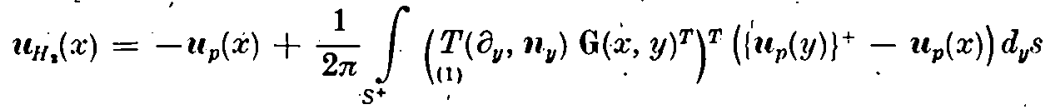

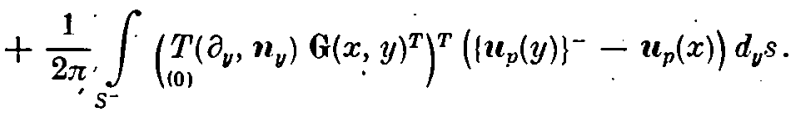


Wegen $\boldsymbol{u}_{p}{ }^{+} \in C^{1,8}\left(D^{+}\right)$ist $\boldsymbol{u}_{p}{ }^{+} \in C^{0,1}\left(D^{+}\right)$und damit ist $\boldsymbol{u}_{p}^{+{ }^{+}}$zusammen mit den Grenzwerten $\left\{u_{p}{ }^{+}(y)\right\}^{+}$in $C^{0.1}\left(\overline{D^{+}}\right)$, also gilt

$$
\left|\left\{u_{p j}(y)\right\}^{+}-u_{p j}(x)\right| \leqq A|y-x| \text { für } y \in S^{+}, x \in D^{+} .
$$

Damit ist. der Integrand des Integrals über $S^{+}$beschränkt, wenn $x \in D^{+}$. Sei nun $x \in K\left(y_{0}, \frac{\delta}{2}\right) \cap D^{+}$und $y \in S^{-} \cap K\left(y_{0}, \delta\right)$. Dann ist

$$
\begin{aligned}
& \mid\left(\begin{array}{l}
\left.T\left(\partial_{y}, n_{y}\right) \mathbf{G}(x, y)^{T}\right)^{T} \cdot\left(\left\{u_{p}(y)\right\}^{-}-u_{p}(x)\right) \mid \\
.
\end{array}\right.
\end{aligned}
$$

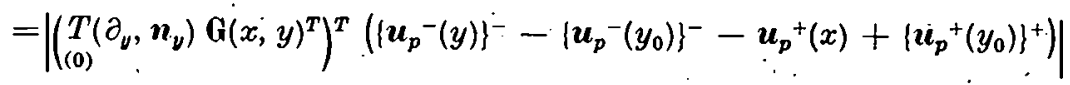

$$
\begin{aligned}
& \leqq C_{1} \frac{\left|y-y_{0}\right|}{|x-y|}+C_{2} \frac{\left|x-y_{0}\right|}{|x-y|} \leqq C_{3} \text {. }
\end{aligned}
$$

Also ist $\boldsymbol{u}_{H_{2}}(x)$ in $D^{+} \cup D^{-}$beschränkt.

Nun ist nach (2.4)

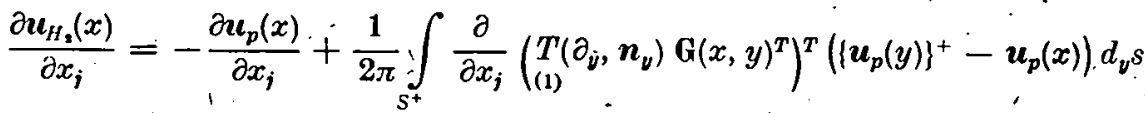

$$
\begin{aligned}
& +\frac{1}{2 \pi} \int_{S^{-}} \frac{\partial}{\partial x_{j}}\left(\underset{(0)}{T}\left(\partial_{y}, n_{\nu}\right) \mathbf{G}(x, y)^{T}\right)^{T}\left(\left\{\boldsymbol{u}_{p}(y)\right\}^{-}-\boldsymbol{u}_{p}(x)\right) d_{y^{s}}
\end{aligned}
$$

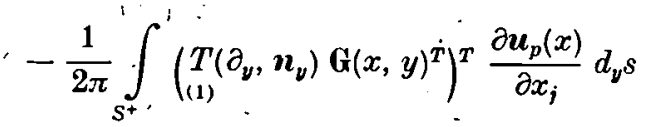

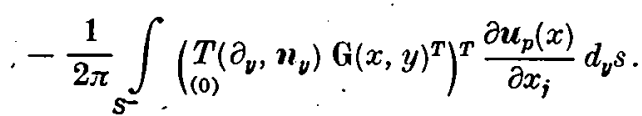

Nach Voraussetzung ist $\frac{\partial u_{p}(x)}{\partial x_{j}}$ in $D^{+} \cup D^{-}$beschränkt. Sei nụn $x \in K\left(y_{0}, \frac{\delta}{2}\right) \cap D$ und $y \in S \cap K\left(y_{0}, \delta\right)$, dann lassen sich die Integranden der Integrale in (2.5) nach oben abschätzen durch $C \cdot|x-y|^{-1}$. Unter Verwendung von (2.2) erhält man leicht

$$
\int_{s \cap K\left(\nu_{\theta} \delta\right)} \frac{1}{|x-y|} d_{\nu} s=\mathcal{O}\left(\ln \cdot \frac{1}{\varrho_{x}}\right) .
$$

Also gilt sicher $\frac{\partial u_{H_{1}}(x)}{\partial x_{j}}=\mathcal{O}\left(\frac{1}{\sqrt{\varrho_{x}}}\right)$.

Es bleibt nun nur noch zu zeigen $\left\{T u_{H_{3}}\left(x_{0}\right)\right\} \in L_{2}^{2}(S)$ : Dazu genügt . es, $\left\{T\left(\partial_{x_{0}}, n_{x_{0}}\right) \dot{u}_{H_{3}}\left(x_{0}\right)\right\}^{+} \in L_{2}{ }^{2}\left(S^{+}\right)$nachzuweisen. Nun ist für $x \in D^{+}$

$T\left(\partial_{x}, \boldsymbol{n}_{x}\right) \boldsymbol{u}_{H},(x)=-T\left(\partial_{x}, n_{x}\right) \boldsymbol{u}_{p}(x)$

(1)

$$
\begin{aligned}
& \text { (1) }
\end{aligned}
$$

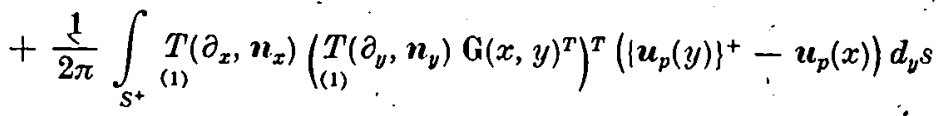




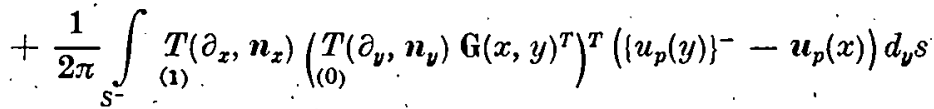

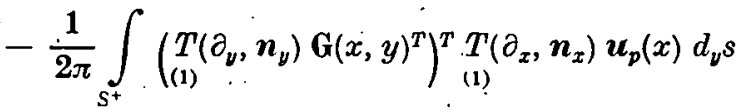

$$
\begin{aligned}
& -\frac{1}{2 \pi} \int_{s^{-}}\left(\frac{T}{(0)}\left(\partial_{y}, \boldsymbol{n}_{y}\right) \mathbf{G}(x, y)^{T}\right)_{(1)}^{T} \underset{(1)}{T}\left(\partial_{x}, \boldsymbol{n}_{x}\right) \dot{u}_{p}(x) d d_{y} s
\end{aligned}
$$

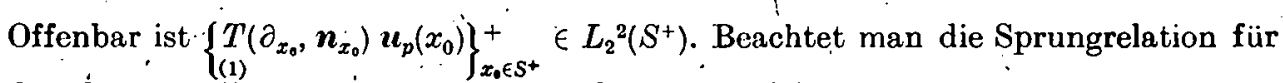
das elastische Potential der.doppelten Schicht, so folgt

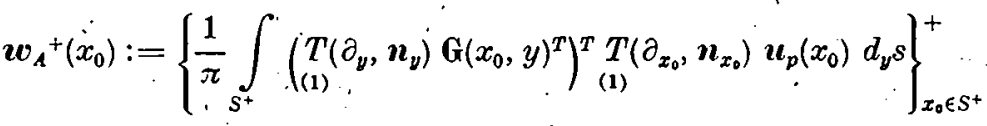

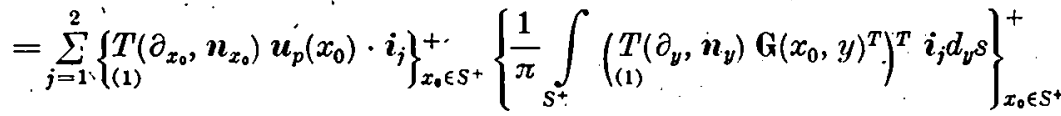

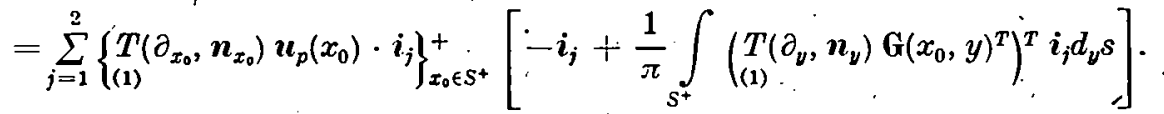

Der Integraloperator in der eckigen Klammer ist ein linearer beschränkter Operator von $L_{2}{ }^{2}\left(S^{\dagger}\right)$ in $L_{2}{ }^{2}\left(S^{+}\right)$(s. Kap. 3) und der Grenzwert davor beschränkt, also ist $w_{A}{ }^{+}\left(x_{0}\right) \in L_{2}^{-2}\left(S^{+}\right)$. Weiter ist

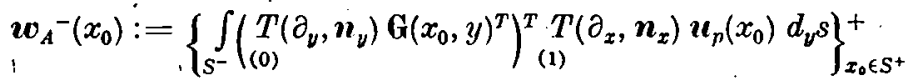

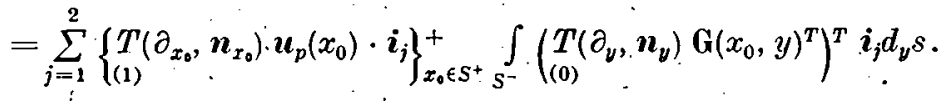

Nun ist

$$
k(y)=\left\{\begin{array}{lll}
0^{\prime} & \text { für } & y \in S^{+} \\
i_{j} & \text { für } & y \in S^{-}
\end{array}\right.
$$

in $L_{2}{ }^{2}(S)$ und $\left.\int_{S}\left(\begin{array}{c}T\left(\partial_{y}^{\prime}\right. \\ ,\end{array} \boldsymbol{n}_{y}\right) \mathbf{G}(x, y)^{T}\right)^{T} \boldsymbol{k}(y) d_{\nu} s$ ein linearer beschränkter Operator von $L_{2}^{2}(S)$ in $L_{2}^{2}(S)$ (s. Kap. 3), also ist $w_{A}^{-}\left(x_{0}\right) \in L_{2}{ }^{2}\left(S^{+}\right)$.

Für $x \in D^{+}, y \in S^{+}$zerlegen wir $\mathbf{G}(x, y)=\Gamma(x, y)+. \mathbf{V}(x, y)$ in die Summe aus dem Somiglianaschen Tensor $\Gamma(x, y)$ und der Kompensatrix $\mathbf{V}(x, y)$ (s. [13]) und setzen $\left\{\boldsymbol{u}_{p}(y)\right\}^{+} \in C^{1, \beta}\left(S^{+}\right)$fort $\mathrm{zu}^{(1)} \boldsymbol{u}_{p}{ }^{*}(y) \in C^{1, \beta}(S)$. Dann ist

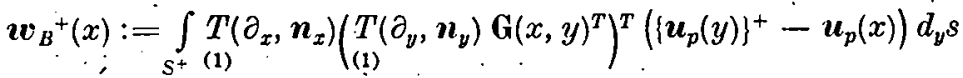

$$
\begin{aligned}
& =\boldsymbol{w}_{B_{1}}^{+}(x)+{ }^{\prime} v_{B_{2}}^{+}(x)+w_{B_{3}}^{+}(x)
\end{aligned}
$$


mit

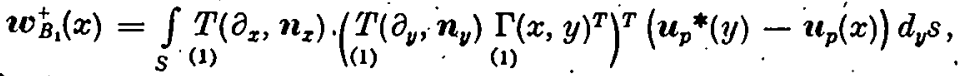

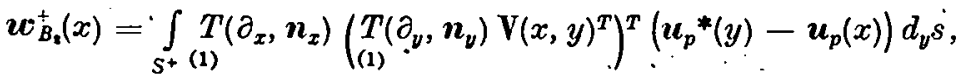

$$
\begin{aligned}
& \boldsymbol{w}_{B_{z}}^{+}(x)=-\int_{S^{-}} T_{(1)}\left(\partial_{x}, n_{x}\right)\left(\underset{(1)}{T\left(\partial_{y}, n_{y}\right)} \underset{(1)}{\Gamma}(x, y)^{T}\right)^{T}\left(u_{p}^{*}(y)-u_{p}(x)\right) d d_{y^{\prime}} s
\end{aligned}
$$

Nun ist $\left\{\boldsymbol{w}_{B_{1}}^{+}\left(\dot{x}_{0}\right)\right\}_{x_{0} \in S}^{+} \in C^{0, \beta}(S) \subset L_{2}{ }^{2}(S)$ (vgl. [16: S. 226]). Weiter ist

also

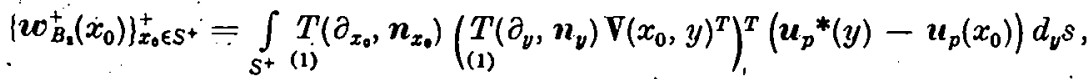

$$
\begin{aligned}
\left|\left\{\boldsymbol{w}_{B_{2} k}^{+}\left(x_{0}\right)\right\}_{x_{0} \in S^{+}}^{+}\right| & \leqq C \int_{s^{+}} \frac{\left|x_{0}-y\right|}{\left(x_{1}^{0}-y_{1}\right)^{2}+\left(x_{2}{ }^{0}+y_{2}\right)^{2}} d_{v^{s}} \\
& \leqq C \int_{s_{+}^{+}} \frac{1}{x_{2}{ }^{0}+y_{2}} d_{y^{\prime}}=\mathcal{O}\left(\left|\ln x_{2}{ }^{0}\right|\right) .
\end{aligned}
$$

Damit ist $\left\{\boldsymbol{w}_{B_{z}}^{+}\left(x_{0}\right)\right\}_{x_{0} \in S^{+}}^{+} \in L_{2}{ }^{2}\left(S^{+}\right) . \therefore$ Bei $\boldsymbol{w}_{B_{3}}^{+}(x)$ kann man den Grenzübergang $x \rightarrow x_{0} \in S^{+}$ebenfalls unter dem Integral ausführen, und man erhält

$$
\left|\left\{\boldsymbol{w}_{B_{3} k}^{*}\left(x_{0}\right)\right\}^{+}\right| \leqq C \int_{S^{-}} \frac{1}{x_{2}{ }^{0}-y_{2}{ }^{2}} d y=\mathcal{O}\left(\left|\ln x_{2}{ }^{0}\right|\right) .
$$

Also ist $\left\{w_{B}^{+}\left(x_{0}\right)\right\}_{x_{0} \in S^{+}}^{+} \in L_{2}^{2}\left(S^{+}\right)$.

Wegen $\left\{\boldsymbol{u}_{p}{ }^{+}\left(y_{0}\right)\right\}^{+}=\left\{\boldsymbol{u}_{p}{ }^{-}\left(y_{0}\right)\right\}^{-}$, kann man schreiben

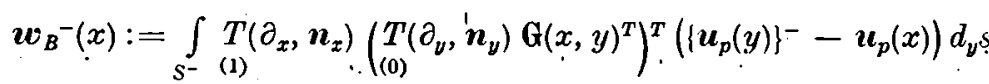

$$
\begin{aligned}
& =\boldsymbol{w}_{B_{1}^{\prime}}^{-}(x)+\boldsymbol{w}_{B_{3}}^{-}(x) \text {. }
\end{aligned}
$$

mit

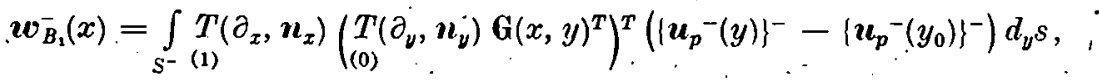

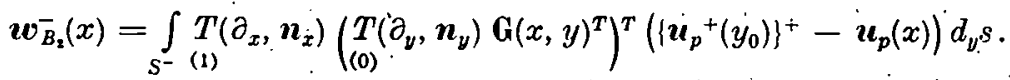

In beiden Integralen kann man den Grenzübergang $x \rightarrow \dot{x}_{0} \dot{\epsilon} S^{+}$unter dem Integral ausführen, und man erhält_in einer Unugebung von $y_{0}$

$$
\begin{aligned}
\cdot\left|\left\{w_{B_{1} k}^{-}\left(x_{0}\right)\right\}^{+}\right| & \leqq C \int_{s^{-}} \frac{\left|y_{0}-y\right|}{\left|x_{0}^{\prime}-y\right|^{2}} d_{y} s \leqq C \int_{s^{-}} \frac{1}{\left|x_{0}-y\right|} d_{y^{s}} \leqq C \int_{s^{-}} \frac{1}{x_{2}{ }^{0}-y_{2}} d_{y^{s}} \\
& =\mathscr{O}\left(\left|\ln x_{2}{ }^{0}\right|\right)
\end{aligned}
$$

und

$$
\left|\left\{w_{B_{2} k}^{-}\left(x_{0}\right)\right\}^{+}\right| \leqq C \int_{S^{-}} \frac{\left|y_{0}-x\right|^{\prime}}{\left|x_{0}-y\right|^{2}} d_{y^{\prime}} s \leqq C \int_{S^{-}} \frac{1}{\left|x_{0}-y\right|} d_{y^{\prime}} s=\mathcal{O}\left(\left|\ln x_{2}^{0}\right|\right)
$$

Also ist $\left\{\boldsymbol{w}_{B_{2}}^{-}\left(x_{0}\right)\right\}_{x_{0} \in S^{+}}^{+} \in L_{2}{ }^{2}\left(S^{+}\right)$. Damit ist Satz 2.5 bewiesen 


\section{Integralgleichungen}

Zur Lösung des Problems II $(D ; 0,0 ; \boldsymbol{P} ; \mathbf{0}, \mathbf{0})$ machen wir den, Ansatz $\boldsymbol{V}(x, \varphi)$ (vgl. (2.1)), der a priori die Differentialgleichung und die homogenen Kontaktbedingungen erfüllt (vgl. Satż 2.4). Die Randbedingung (1.2) führt auf Grund der Sprungrelation für das elastische Potential der einfachen Schicht (vgl. [18: (4.2)]) auf die Integralgleichung

$$
\mathscr{A} \varphi:=\varphi(x)+\frac{1}{\pi} \int_{5} \mathbf{K}(x, y) \varphi(y) d_{y^{s}}=\boldsymbol{P}(x)
$$

mit

$$
\mathbf{K}(x, y)= \begin{cases}T\left(\partial_{x}, \boldsymbol{n}_{x}\right) \mathbf{G}(x, y) & \text { für } x \in S^{+} \\ \underset{(1)}{T\left(\partial_{x}, n_{x}\right) \mathbf{G}(x, y)} & \text { für } x \in S^{-}\end{cases}
$$

Für $x \in S^{+}, y \in S^{+}$ist

mit

$$
\mathbf{K}(x, y)=\mathbf{S}(x, y)+\mathbf{F}(x, y)
$$

$$
\begin{aligned}
\mathbf{S}(x, y)= & -\frac{\mu_{1}}{\lambda_{1}+2 \mu_{1}} \sum_{j=1}^{2} n_{j}(x) \frac{x_{j}-y_{j}}{|x-y|^{2}}\left(\begin{array}{ll}
1 & 0 \\
0 & 1
\end{array}\right) \\
& +\frac{\mu_{1}}{\lambda_{1}+2 \mu_{1}}\left(n_{1}(x) \frac{x_{2}-y_{2}}{|x-y|^{2}}-n_{2}(x) \frac{x_{1} \div y_{1}}{|x-y|^{2}}\right)\left(\begin{array}{rr}
0 & 1 \\
-1 & 0
\end{array}\right) \\
& -\frac{2\left(\lambda_{1}+\mu_{1}\right)}{\lambda_{1}+2 \mu_{1}} \sum_{j=1}^{2} n_{j}(x) \frac{x_{j}-y_{j}}{|x-y|^{4}}(x-y)(x-y)^{T}
\end{aligned}
$$

und

$$
\begin{aligned}
& \mathbf{F}(x, y)={ }^{\prime}+\frac{\ddot{x}_{1}-y_{1}}{\left(x_{1}-y_{1}\right)^{2}+\left(x_{2}+{ }^{-} y_{2}\right)^{2}}\left(\begin{array}{ll}
K_{1}{ }^{+} n_{1}(x) & K_{2}{ }^{+} n_{2}(x) \\
K_{3}{ }^{+} n_{2}(x) & K_{2}{ }^{+} n_{1}(x)
\end{array}\right) \\
& +\frac{x_{2}}{\left(x_{1}-y_{1}\right)^{2}+\left(x_{2}+y_{2}\right)^{2}}\left(\begin{array}{ll}
K_{2}{ }^{+} n_{2}(x) & K_{3}{ }^{+} n_{1}(x) \\
K_{2}+n_{1}(x) & K_{1}{ }^{+} n_{2}(x)
\end{array}\right) \\
& +\frac{y_{2}}{\left(x_{1}-y_{1}\right)^{2}+\left(x_{2}+y_{2}\right)^{2}}\left(\begin{array}{cc}
K_{4}{ }^{+} n_{2}(x) & K_{5}{ }^{+} n_{1}(x) \\
\dot{K}_{4}{ }^{+} n_{1}(x) & K_{6}{ }^{+} n_{2}(x)
\end{array}\right) \\
& +\frac{x_{2}\left(x_{1}-y_{1}\right)^{2}}{\left[\left(x_{1}-y_{1}\right)^{2}+\left(x_{2}+y_{2}\right)^{2}\right]^{2}} 4 \mu_{1} H_{9}+\left(\begin{array}{ll}
-n_{2}(x) & -n_{1}(x) \\
-n_{1}(x) & +n_{2}(x)
\end{array}\right) \\
& +\frac{y_{2}\left(x_{1 .}-y_{1}\right)^{2}}{\left[\left(x_{1}-y_{1}\right)^{2}+\left(x_{2}+y_{2}\right)^{2}\right]^{2}}\left(\begin{array}{ll}
2 \mu_{1} H_{11}^{+} \dot{n}_{2}(x) & K_{i}{ }^{+} n_{1}(x) \\
2 \mu_{1} H_{11}^{+} n_{1}(x) & K_{3}{ }^{+} n_{2}(x)
\end{array}\right) \\
& +\frac{x_{2}\left(x_{1}-y_{1}\right)\left(x_{2}+y_{2}\right)}{\left[\left(x_{1}-y_{1}\right)^{2}+\left(x_{2}+y_{2}\right)^{2}\right]^{2}} 4 \mu_{1} H_{9}+\left(\begin{array}{ll}
+n_{1}(x) & -n_{2}(x) \\
-n_{2}(x) & -n_{1}(x)
\end{array}\right) \\
& +\frac{y_{2}\left(x_{1}-y_{1}\right)\left(x_{2}+y_{2}\right)}{\left[\left(x_{1}-y_{1}\right)^{2}+\left(x_{2}+y_{2}\right)^{2}\right]^{2}}\left(\begin{array}{ll}
K_{7}+n_{1}(x) & -2 \mu_{1} H_{11}^{+} n_{2}(x) \\
K_{8}{ }^{+} n_{2}(x) & -2 \mu_{1} H_{11}^{+} n_{1}(x)
\end{array}\right) \\
& +\frac{x_{2} y_{2}\left(x_{1}-y_{1}\right)}{\left[\left(x_{1}-y_{1}\right)^{2}+\left(x_{2}+y_{2}\right)^{2}\right]^{2}} 12 \mu_{1} H_{11}^{+}\left(\begin{array}{l}
+n_{1}(x)+\dot{n}_{2}(x) \\
-n_{2}(x)+n_{1}(x)
\end{array}\right)
\end{aligned}
$$


mit

$$
\begin{aligned}
& +\frac{x_{2} y_{2}\left(x_{2}+y_{2}\right)}{\left[\left(x_{1}-y_{1}\right)^{2}+\left(x_{2}+y_{2}\right)^{2}\right]^{2}} 4 \mu_{1} H_{11}^{+}\left(\begin{array}{l}
+n_{2}(x)-n_{1}(x) \\
+n_{1}(x)+n_{2}(x)
\end{array}\right) \\
& +\frac{x_{2} y_{2}\left(x_{1}-y_{1}\right)^{3}}{\left[\left(x_{1}-y_{1}\right)^{2}+\left(x_{2}+y_{2}\right)^{2}\right]^{3}} 16 \mu_{1} H_{11}^{+}\left(\begin{array}{l}
-n_{1}(x)-n_{2}(x) \\
+n_{2}(x) .-n_{1}(x)
\end{array}\right) \\
& +\frac{x_{2} y_{2}\left(x_{1}-y_{1}\right)^{2}\left(x_{2}+y_{2}\right)}{\left[\left(x_{1}-y_{1}\right)^{2}+\left(x_{2}+y_{2}\right)^{2}\right]^{3}} 16 \mu_{1} H_{11}^{+}\left(\begin{array}{l}
-n_{2}(x)+n_{1}(x) \\
-n_{1}(x)-n_{2}(x)
\end{array}\right)
\end{aligned}
$$

$$
\begin{aligned}
& K_{1}{ }^{+}=-\left(\lambda_{1}+2 \mu_{1}\right) H_{6}{ }^{+}-\lambda_{1} H_{8}{ }^{+}+\lambda_{1} H_{9}{ }^{+}, \\
& K_{2}{ }^{+}=\mu_{1}\left(-H_{6}{ }^{+}+H_{8}{ }^{+}+\dot{H}_{9}^{+}\right) \text {, } \\
& K_{3}{ }^{+}=-\lambda_{1} H_{6}{ }^{+}-\left(\lambda_{1}+2 \mu_{1}\right) H_{8}{ }^{+}+\left(\lambda_{1}+2 \mu_{1}\right) H_{9}{ }^{+} \text {, } \\
& K_{4}{ }^{+}=\mu_{1}\left(-H_{6}{ }^{+}+H_{8}{ }^{+}-H_{9}{ }^{+}-H_{11}^{+}\right) \text {, } \\
& K_{5}{ }^{+}=-\lambda_{1} H_{6}{ }^{+}-\left(\lambda_{1}+2 \mu_{1}\right) H_{8}{ }^{+}-\left(\lambda_{1}+2 \mu_{1}\right) H_{9}{ }^{+}-\lambda_{1} H_{11}^{+} \text {, } \\
& K_{6}{ }^{+}=-\left(\lambda_{1}+2 \mu_{1}\right) H_{6}{ }^{+}-\lambda_{1} H_{8}{ }^{+}-\lambda_{1} H_{9}{ }^{+}-\left(\lambda_{1}+2 \mu_{1}\right) H_{11}^{+} \text {, } \\
& K_{7}^{+}=4\left(\lambda_{1}+\mu_{1}\right) H_{9}^{+}+2 \lambda_{1} H_{11}^{+}, \quad K_{8}{ }^{+}=K_{7}^{+}+4 \mu_{1} H_{11}^{+} .
\end{aligned}
$$

Für $x \in S^{-}, y \in S^{-}$erhält man $K(x, y)$ aus (3:3), indem man $\mu_{1}$ durch $\mu_{0}, \lambda_{1}$ durch $\lambda_{0}, H_{9}{ }^{+}, H_{11}^{+}$durch $H_{9}^{-}, H_{11}^{-}$und $K_{1}^{+}, \ldots, K_{8}+$ durch $K_{1}^{-}, \ldots, K_{8}^{-}$ersetzt. Dabei geht $K_{j}^{-}(j=1, \ldots, 8)$ aus $K_{j}^{+}$durch die Ersetzungen $\lambda_{1}\left|\lambda_{0}, \mu_{1}\right| \mu_{0}, H_{k}+\mid H_{k}-$ hervor. Die Konstanten $H_{k}{ }^{+}, H_{k}{ }^{-}(k=1, \ldots, 11)$ sind als Funktion der Laméschen Moduln $\lambda_{0}, \mu_{0}, \lambda_{1}, \mu_{1}$ in [13] explizit angegeben. Als Funktiòn der Youngschen Moduln $E_{0}, E_{1}$ und der Poissonschen Zahlen $\nu_{0}, \nu_{1}$ sind sie am Ende der Arbeit aufgeschrieben..

Für $x \in S^{+}, y \in S^{-}$ist

$$
\begin{aligned}
& \mathbf{K}(x, y) \doteq \mathbf{F}(x, y)=\frac{x_{1}-y_{1}}{\left(x_{1}-y_{1}\right)^{2}+\left(x_{2}-y_{2}\right)^{2}}\left(\begin{array}{ll}
K_{9}{ }^{-} n_{1}(x) & K_{\mathrm{i0}}^{-} n_{2}(x) \\
K_{11}^{-} n_{2}(x) & K_{10}^{-} n_{1}(x)
\end{array}\right) \\
& +\frac{x_{2}-y_{2}}{\left(x_{1}-y_{1}\right)^{2}+\left(x_{2}-y_{2}\right)^{2}}\left(\begin{array}{ll}
K_{12}^{-} n_{2}(x) & K_{13}^{-} n_{1}(x) \\
K_{12}^{-} n_{1}(x) & K_{14}^{-} n_{2}(x)
\end{array}\right) \\
& +\frac{x_{2} H_{4}^{-}+y_{2} H_{5}^{-}}{\left(x_{1}-y_{1}\right)^{2}+\left(x_{2}-y_{2}\right)^{2}} 2 \mu_{1}\left(\begin{array}{ll}
-n_{2}(x) & -n_{1}(x) \\
-n_{1}(x) & +n_{2}(x)
\end{array}\right) \\
& +\frac{\left(x_{2} H_{4}^{-}+y_{2} H_{5}^{-}\right)\left(x_{1}-y_{1}\right)^{2}}{\left[\left(x_{1}-y_{1}\right)^{2}+\left(x_{2}-y_{2}\right)^{2}\right]^{2}} 4 \mu_{1}\left(\begin{array}{cc}
+n_{2}(x) & +n_{1}(x) \\
+n_{1}(x) & -n_{2}(x)
\end{array}\right)
\end{aligned}
$$

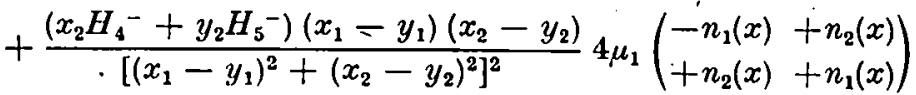

mit

$$
\begin{aligned}
& K_{9}^{-}=-\left(\dot{\lambda}_{1}+2 \mu_{1}\right) H_{1}^{-}+\lambda_{1} H_{3}^{-}-\lambda_{1} H_{4}^{-}, K_{10}^{-}=\mu_{1}\left(-H_{1}^{-}-H_{3}^{-}-H_{4}^{-}\right), \\
& K_{11}^{-}=-\lambda_{1} H_{1}^{-}+\left(\lambda_{1}+2 \mu_{1}\right) H_{3}^{-}-\left(\lambda_{1}+2 \mu_{1}\right) H_{4^{-}}, K_{12}^{-}=K_{10}^{-}+2 \mu_{1} H_{4}^{-}, \\
& K_{13}^{-}=K_{11}^{-}+2 \mu_{1} H_{4}^{-}, K_{14}^{-}=K_{9}^{-}-2 \mu_{1} H_{4}^{-} .
\end{aligned}
$$

Analog zu oben erhält man $\mathbf{K}(x, y)$ für $x \in S^{-}, y \in S^{+}$aus (3.4) durch die Ersetzungen $\mu_{1}\left|\mu_{0}, H_{4}^{-}\right| H_{4}^{+}, H_{5}^{-}\left|H_{5}^{+}, K_{9}^{-}, \ldots, K_{14}^{+}\right| K_{9}^{+}, \ldots, K_{14}^{+}$. Dabei ergibt sich $K_{j}^{+}(j=9, \ldots, 14)$ aus $K_{j}^{-}$durch die Ersetzungen $\lambda_{1}\left|\lambda_{0}, \mu_{1}\right| \mu_{0}, H_{k}^{-} \mid H_{k}{ }^{+}$.

Neben dem singulären Kern $\mathbf{S}(x, y)$ tritt der Kern $\mathbf{F}(x, y)$ auf, der feststehende Singularitäten bei $x=y=y^{+}( \pm 1)$ hat. 
Wir transformieren jetzt das System (3.1) mit Hilfe der Parameterdarstellung von $S^{+}$und $S^{-}$auf das Intervall $[-1,+1]$, indem wir setzen:,

$$
\begin{aligned}
\Phi(t)= & \left(\begin{array}{l}
\Phi_{1}(t) \\
\Phi_{2}(t) \\
\Phi_{3}(t) \\
\Phi_{4}(t)
\end{array}\right)=\left(\begin{array}{l}
\varphi_{1}\left(y^{+}(t)\right) \\
\varphi_{2}\left(y^{+}(t)\right) \\
\varphi_{1}\left(y^{-}(t)\right) \\
\varphi_{2}\left(y^{-}(t)\right)
\end{array}\right), p(t)=\left(\begin{array}{l}
p_{1}(t) \\
p_{2}(t) \\
p_{3}(t) \\
p_{4}(t)
\end{array}\right) \doteq\left(\begin{array}{l}
P_{1}\left(y^{+}(t)\right) \\
P_{2}\left(y^{+}(t)\right) \\
P_{1}\left(y^{-}(t)\right) \\
P_{2}\left(y^{-}(t)\right)
\end{array}\right), \\
k(t, \tau)= & \left(k_{i j}(t, \tau)\right)_{i_{j}=1, \ldots, 4}: \\
= & \left(\begin{array}{ll}
\mathbf{K}\left(y^{+}(t), y^{+}(\tau)|| \dot{y}^{+}(\tau) \mid,\right. & \mathbf{K}\left(y^{+}(t), y^{-}(\tau)\right)\left|\dot{y}^{-}(\tau)\right| \\
\mathbf{K}\left(y^{-}(t), y^{+}(\tau)\right)\left|\dot{y}^{+}(\tau)\right| & \mathbf{K}\left(y^{-}(t), y^{-}(\tau)\right) \mid \dot{y^{-}(\tau) \mid}
\end{array}\right)
\end{aligned}
$$

Dann geht (3.1) iiber in (beachte Satz 1.2, S. 36 in [20])

$$
\mathscr{B} \dot{\Phi}:=\Phi(t)+\frac{\cdot 1}{\pi} \int_{-1}^{+1} k(t, \tau) \Phi(\tau) d \tau=p(t)
$$

Mit Ergebnissen in [22: Kap.I, §7] folgt unter der Voraussetzung $S^{+} \subset C^{1, \alpha}$, $S^{-}=C^{1 . \alpha}$

$$
\begin{aligned}
k(t ; \tau)= & \left(\alpha_{i j}\right) \frac{1}{t-\tau} \\
\quad & +\left(\begin{array}{cccc}
h_{11}(t, \tau) & h_{12}(t, \tau) & 0 & 0 \\
h_{21}(t, \tau) & h_{22}(t, \tau) & 0 & 0 \\
0 & 0 & h_{33}(t, \tau) & h_{34}(t, \tau) \\
0 & 0 & h_{43}(t, \tau) & h_{44}(t, \tau)
\end{array}\right) \frac{1}{t-\tau}+f(t, \tau)
\end{aligned}
$$

$\cdot \mathrm{mit}$

$$
\left(\alpha_{i j}\right)=\left[\begin{array}{cccc}
0 & -\frac{\mu_{1}}{\lambda_{1}+2 \mu_{1}} & 0 & 0 \\
\frac{\mu_{1}}{\lambda_{1}+2 \mu_{1}} & 0 & 0 & 0 \\
0 & 0 & 0 & \frac{\mu_{0}}{\lambda_{0}+2 \mu_{0}} \\
0 & 0 & -\frac{\mu_{0}}{\lambda_{0}+2 \mu_{0}} & 0
\end{array}\right] .
$$

Dabei ist $h_{i j}(t, \tau) \in H^{\alpha}([-1,+1] \times[-1,+1])$ (s. [20: S. 33]), $h_{i j}(t, t)=0$ und $f(t, \tau)$ hat feststehende Singularitäten bei $(t, \tau)=(1,1)$ und $(t, \tau)=(-1,-1)$. Die Elemente $f_{i j}(t, \tau)$ von $f(t, \tau)$, zerlegen wir in

$$
f_{i j}(t, \tau)=h^{+}(\tau) f_{i j}(t, \tau)+h^{-}(\tau) f_{i j}(t, \tau) \text {. }
$$

Dabei sind $h^{+}(\tau), h^{-}(\tau) \in C^{\infty}([-1,+1]), h^{ \pm}(\tau) \geqq 0, h^{+}(\tau)=1$ für $\tau \in\left[\frac{1}{2}, 1\right]$, $h^{+}(\tau)=0 \quad$ für $\quad \tau \in\left[-1,-\frac{1}{2}\right], h^{-}(\tau)=0$ für $\quad \tau \in\left[\frac{1}{2}, 1\right], h^{-}(\tau)=1$ für $\tau \in\left[-1,-\frac{1}{2}\right]$ und $h^{+}(\tau)+h^{-}(\tau)=1$ für $\tau \in[-1,+1]$. Dann hat $h^{+}(\tau) \dot{f_{i j}}(t, \tau)$ nur bei $(t, \tau)=(1,1)$ und $h^{-}(\tau) f_{i j}(t, \tau)$ nur bei $(t, \tau)^{\prime}=(-1,-1)$ eine Singularität. 
Bei der Normierung $\dot{y}_{2}^{ \pm}(1)=\mp 1, \dot{y}_{2} \pm(-1)= \pm 1$ erhält man für die Gleichung der Tángente an $S \pm$ im Punkt $y^{+}(1)$

$$
\begin{aligned}
& \dot{y}_{1}^{ \pm}(t)_{+1}=y_{1}^{ \pm}(1)+\cot \alpha_{1}^{ \pm}(t-1),\left(y_{1}^{+}(1)=y_{1}{ }^{-}(1)\right) \\
& \grave{y}_{2}^{ \pm}(t)_{+1}= \pm(1-t)
\end{aligned}
$$

und im Punkt $y^{+}(-1)$

$$
\begin{aligned}
& \check{y}_{1}^{ \pm}(t)_{-1}=y_{1}^{ \pm}(-1)+\cot \alpha_{-1}^{ \pm}(t+1),\left(y_{1}^{+}(-1)=y_{1}{ }^{-}(-1)\right) \\
& \check{y}_{2}^{ \pm}(t)_{-1}= \pm(t+1) .
\end{aligned}
$$

Hierbei sind $\alpha_{1}{ }^{+}, \alpha_{1}^{-}$die Winkel der Tangente an $S^{+}, S^{-}$mit der $x_{1}$-Achse im Punkt $y^{+}(1)$ und $\alpha_{-1}^{+}, \alpha_{-1}^{-}$die entsprechenden Winkél im Punkt $y^{+}(-1)$.

Führen wir die Bezeichnungen ein $(i, j=1,2)$ :

$$
\begin{aligned}
\therefore & f_{i j}^{0 \pm}(t, \tau)=F_{i j}\left(\dot{y}^{+}(t)_{ \pm 1}, \dot{y}^{+}(\tau)_{ \pm 1}\right)\left|\dot{y}^{+}( \pm 1)\right|, \\
& f_{i, j+2}^{0 \pm \pm}(t, \tau)=F_{i j}\left(\dot{y}^{+}(t)_{ \pm 1}, \dot{y}^{-}(\tau)_{ \pm 1}\right)\left|\dot{y}^{-}( \pm 1)\right|, \\
& f_{i+2, j}^{0 \pm}(t, \tau)=F_{i j}\left(\dot{y}^{-}(t)_{ \pm 1}, \dot{y}^{+}(\tau)_{ \pm 1}\right)\left|\dot{y}^{+}( \pm 1)\right|, \\
& f_{i+2, j+2}^{0 \pm}(t, \tau)=F_{i j}\left(\dot{y}^{-}(t)_{ \pm 1}, \dot{y}^{-}(\tau)_{ \pm 1}\right) .\left|\dot{y}^{-}( \pm 1)\right|
\end{aligned}
$$

dann erhält man durch eine genaue Analyse aus (3.7) $(i, j=1, \ldots, 4)$ :

$$
\begin{aligned}
& f_{i j}(t, \tau)=f_{i j}^{0+}(t, \tau)+f_{i j}^{0-(t, \tau)}+\frac{m_{i j}^{\bar{\tau}}(t, \tau)}{(1-t)^{1-\alpha}+(1-\tau)^{1-\alpha}} \\
& \quad+\frac{m_{i j}(t, \tau)}{(1+t)^{1-\alpha}+(1+\tau)^{1-\alpha}},
\end{aligned}
$$

wobei $m_{i j}^{+}(t, \tau)$ für $(t, \tau) \neq(1,1)$ und $\ddot{m}_{i j}(t, \tau)$ für $(t, \tau) \neq(-1,-1)$ stetig und beschränkt ist. Für die Kerne $F_{i j}(x, y)$ gilt offenbar die Abschätzung

$$
\left|F_{i j}(x, y)\right| \leqq C \frac{1}{\left|x_{2}\right|+\left|y_{2}\right|}
$$

Hieraus folgt

$$
\left|f_{i j}^{0+}(t, \tau)\right| \leqq C^{+} \frac{1}{2-t-\tau}, \cdots\left|f_{i j}^{0-}(t, \tau)\right| \leqq C^{-} \frac{1}{2+t+\tau}
$$

Aus bekannten Resultaten (s. [3: S. 100, S. 85]) schließt man nun auf folgende Aussage.

Satz 3.1: Sei $S^{+} \subset C^{1, \alpha}, S^{-} \subset C^{1, \alpha}(0<\alpha \leqq 1), \alpha_{1}^{ \pm} \neq 0, \alpha_{-1}^{ \pm} \neq 0$. Dann ist $\mathscr{B}$ in (3.5) ein linearer beschränkter Operator von $L_{2}{ }^{4}([-1,+1])$ in $L_{2}{ }^{4}([-1 ;+1])$ und damit $\mathscr{A}$ in (3.1) ein linearer beschränkter Operator von $L_{2}{ }^{2}(S)$ in $L_{2}{ }^{2}(S)$.

Der zu $\mathscr{A}$ adjungierte Operator $\mathscr{A}^{*}$ ist

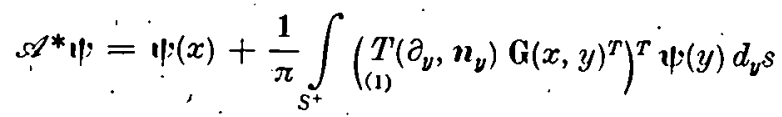

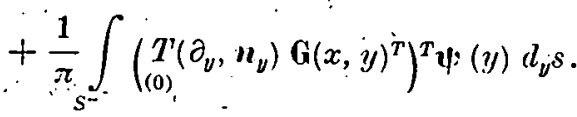


Transformation von $\mathscr{A}^{*}$ auf das Intervall $[-1,+1]$ ergibt für

$$
\begin{aligned}
& \Psi(t)=\left(\begin{array}{l}
\Psi_{1}(t) \\
\Psi_{2}(t) \\
\Psi_{3}(t) \\
\Psi_{4}(t)
\end{array}\right)=\left(\begin{array}{l}
\psi_{1}\left(y^{+}(t)\right) \\
\psi_{2}\left(y^{+}(t)\right) \\
\psi_{1}\left(y^{-}(t)\right) \\
\psi_{2}\left(y^{-}(t)\right)
\end{array}\right) \\
& \mathscr{B}^{T} \Psi=\Psi(t)+\frac{1}{\pi} \int_{-1}^{+1} k^{T}(t, \tau) \Psi(\tau) d \tau
\end{aligned}
$$

mit $\overline{(i, j}=1,2)$

$$
\begin{aligned}
& k_{i j}^{T}(t, \tau)=k_{j i}(\tau, t) \frac{\left|\dot{y}^{+}(\tau)\right|}{\left|\dot{y}^{+}(t)\right|}, \quad k_{i, j \div 2}^{T}(t, \tau)=k_{j+2, i}(\tau, t) \frac{\left|\dot{y}^{-}(\tau)\right|}{\left|\dot{y}^{+}(t)\right|} \\
& k_{1+2, j}^{T}(t, \tau)=k_{j, i+2}(\tau, \ell) \frac{\left|\dot{y}^{+}(\tau)\right|}{\left|\dot{y}^{-}(t)\right|}, k_{i+2, j+2}^{T}(t, \tau)=k_{j+2, i+2}(\tau, t) \frac{\left|\dot{y}^{-}(\tau)\right|}{\left|\dot{y}^{-}(t)\right|}
\end{aligned}
$$

Der zu $\mathscr{B}$ adjungierte Operator ist

$$
\mathscr{B} * \Psi=\Psi(t)+\frac{1}{\pi} \int_{-1}^{+1} k^{*}(t, \tau) \Psi(\tau) d \tau
$$

mit

$$
k_{i j}^{*}(t ; \tau)=k_{j i}(\tau, t)
$$

Satz 3.2: Unter den Voraussetzungen des Satzes 3.1 gilt, daß die starren Verschiebungen $\boldsymbol{a}_{1}, \boldsymbol{a}_{2}, \boldsymbol{a}_{3}$ zu $N\left(\mathscr{A}^{*}\right)$. (Nullraum von $\left.\dot{A}^{*}\right)$ gehören. Entsprechend gilt

$$
\Psi_{(k)}(t)=\left(\begin{array}{l}
a_{k}\left(y^{+}(t)\right) \\
a_{k}\left(y^{-}(t)\right)
\end{array}\right) \subset N\left(\mathscr{B}^{T}\right)
$$

und

$$
\Psi_{(k)}^{*}(t)=\left(\begin{array}{l}
a_{k}\left(y^{+}(t)\right)\left|\dot{y}^{+}(t)\right| \\
a_{k}\left(y^{-}(t)\right)\left|\dot{y}^{-}(t)\right|
\end{array}\right) \subset N\left(\mathscr{B}^{*}\right), \quad k=1,1,3
$$

Beweis: Wendet man die Bettische Formel (s. [18: (3.13)]) an auf $D^{+} \backslash K\left(x_{0}, \varepsilon\right)$, $x_{0} \in S^{+}$und $D^{-}$mit $\boldsymbol{u}=\boldsymbol{u}(y)=\mathbf{G}_{(k)}\left(x_{0}, y\right)$ und $\boldsymbol{v}=\boldsymbol{v}(y)=\boldsymbol{a}_{i}(y)$, dann folgt (beachte [18: S. 13]. und die Symmetrie von $G(x, y)[13]) \mathscr{A}^{*} a_{i}\left(x_{0}\right)=0$ für $x_{0} \in S^{+}$. Analog zeigt $\operatorname{man} \mathscr{A}^{*} a_{\mathrm{i}}\left(x_{0}\right)=0$ für $x_{0} \in S^{-}$

Zum Nachweis, daß $\mathscr{L}\left\{a_{1}, \boldsymbol{a}_{2}, a_{3}\right\}=N\left(\mathscr{A}^{*}\right)$ gilt, benötigen wir folgenden Satz.

Satz 3.3 (Regularitätssatz): Es seien die Voraussetzungen des Satzes 3.1 erfǘllt $\boldsymbol{P}(x) \in L_{2}{ }^{2}(S) \cap C^{0, \beta}\left(S \backslash K_{\varepsilon}\right)$ für jedes $\varepsilon>0,0<\beta<\alpha \leqq 1$, und $\varphi(x) \in L_{2}{ }^{2}(S)$ eine Lösung von $\mathscr{A} \varphi=P$. Dann gilt $\varphi(x) \in C^{0, \beta}\left(S \backslash K_{c}\right)$ für jedes $\varepsilon>0$.

Beweis: Die Anteile in der Integralgleichung, die von der Kompensatrix $\mathrm{V}(x, y)$ herrühren, sind für $\left|x_{2}\right| \neq 0$ gutartig und genügen auf $S \backslash K_{c}$ einer $\boldsymbol{H}$-Bedingung mit dem Exponenten 1. Bringen wir diese auf die rechte Seite, gewinnt $\mathscr{A} \varphi=\boldsymbol{P}$ 
die Giestalt

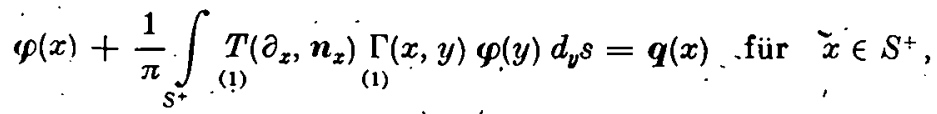

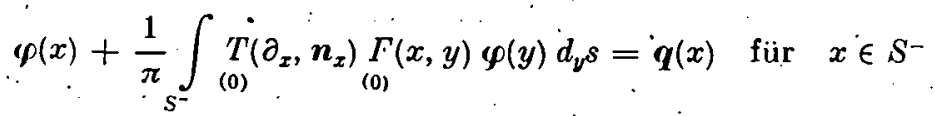

mit $\boldsymbol{q}(x) \in L_{2}{ }^{2}(S) \cap C^{0, \beta}\left(S \backslash K_{c}\right)(\beta=1$, wenn $\boldsymbol{P}(x)=0)$. Es genügt offenbar, den weiteren Beweis für.(3.16) zu führen. Wir transformieren.(3.16) auf das .Intervall $[-1,+1]$ und erhalten

$$
\begin{aligned}
& \Phi_{1}(t)+\frac{\mu_{1}}{\lambda_{1}+2 \mu_{1}} \frac{1}{\pi} \int_{-1}^{+1} \frac{1}{\tau-t} \Phi_{2}(\tau) d \tau+\sum_{j=1}^{2} \frac{1}{\pi} \int_{-1}^{+1} d_{1 j}(t, \tau) \Phi_{j}(\tau) d \tau=q_{1}(t), \\
& \Phi_{2}(t)-\frac{\mu_{1}}{\lambda_{1}+2 \mu_{1}} \frac{1}{\pi} \int_{-1}^{+1} \frac{1}{\tau-t} \Phi_{1}(\tau) d \tau+\sum_{j=1}^{2} \frac{1}{\pi} \int_{-1}^{+1} d_{2 j}(t, \tau) \Phi_{j}(\tau) d \tau=q_{2}(t), \\
& d_{i j}(t, \tau)=\frac{h_{i j}(t, \tau)}{t-\tau}=\frac{h_{i j}(t, \tau)-h_{i j}(t, t)}{t-\tau}, \\
& h_{i j}(t, \tau) \in H^{a}([-1,+1] \times[-1,+1]), \\
& \Phi_{i}(t) \in L_{2}([-1,+1]), q_{i}(t) \in L_{2}^{\prime}([-1,+1]) \cap C^{0, \beta}([-1+\varepsilon, 1-\varepsilon])
\end{aligned}
$$

Wir setzen jetzt das Intervall $[-1,+1] z u$ einer genügend glatten geschlossenen Kurve $L$ fort und betrachten

$$
\begin{aligned}
& \dot{\Phi}(t)= \begin{cases}\boldsymbol{\phi}(t) & \text { für } t \in[-1,+1], \\
0 & \text { für } t \in L \backslash[-1,+1],\end{cases} \\
& \dot{d}_{i j}(t, \tau) \doteq \frac{\hat{h}_{i j}(t, \tau)-\hat{h}_{i j}(t, t)}{t-\tau} \text { für } t, \tau \in L,
\end{aligned}
$$

wobei $\hat{h}_{i j}(t, \tau) \in \dot{H}^{a}(L \times L)$ eine Fortsetzung von $h_{i j}(t, \tau)$ auf $L \times L$ ist (zur Fortsetzbarkeit s. Kap. 4, man mu $B$ noch mit einer genügend glatten Abschneidefunktion multiplizieren),

$$
\begin{aligned}
& \dot{q_{1}}(t)=\left\{\begin{array}{l}
q_{1}(t) \text { für } t \in[-1,+1] ; \\
\frac{1}{\pi} \frac{\mu_{1}}{\dot{\lambda}_{1}+2 \mu_{1}} \int_{-1}^{+1} \frac{1}{\tau-t} \Phi_{2}(\tau) d \tau+\sum_{j=1}^{2} \frac{1}{\pi} \int_{-1}^{+1} d_{1 j}(t, \tau) \Phi_{j}(\tau) d \tau \\
\text { für } t \in L \backslash[-1,+1] ;
\end{array}\right. \\
& \hat{q}_{2}(t)=\left\{\begin{array}{l}
q_{2}(t) \text { für } t \in[-1,+1], \\
-\frac{1}{\pi} \frac{\dot{\mu}_{1}}{\lambda_{1}+2 \mu_{1}} \int_{-1}^{+1} \frac{1}{\tau-t} \Phi_{1}(\tau) d \tau+\sum_{j=1}^{2} \frac{1}{\pi} \int_{-1}^{+1} d_{2 j}(t, \tau) \Phi_{j}(\tau) d \tau
\end{array}\right.
\end{aligned}
$$


Setzen wir noch

$$
\mathscr{S}_{u}=\frac{1}{\pi i} \int_{L} \frac{u(\tau)}{\tau-t} d \tau, \quad \mathscr{V}_{i j} u=\frac{1}{\pi} \int_{L} \hat{d}_{i j}(t, \tau) u(\tau) d \tau
$$

dann kann (3.17) geschrieben werden

$$
\begin{aligned}
& \hat{\Phi}_{1}+i \frac{\mu_{1}}{\hat{\lambda}_{1}+2 \mu_{1}} \mathscr{S} \hat{\Phi}_{2}+\mathscr{V}_{11} \hat{\phi}_{1}+\mathscr{V}_{12} \hat{\phi}_{2}=\hat{q}_{1}, \\
& \hat{\Phi}_{2}-i \frac{\mu_{1}}{\hat{\lambda}_{1}+2 \mu_{1}} \mathscr{S} \hat{\Phi}_{1}+\grave{\mathscr{V}}_{21} \hat{\phi}_{1}+\mathscr{V}_{.22} \hat{\Phi}_{2}=\hat{q}_{2}
\end{aligned}
$$

mit $\hat{q}_{i} \in L_{2}(L) \cap C^{0,8}(L \backslash(K(1, \varepsilon) \cup K(-1 ; \varepsilon)))$. Jetzt wird (3.18) von links mit

$$
\left(\begin{array}{ccc}
a \mathscr{I} & - & -i \frac{\mu_{1}}{\lambda_{1}+2 \mu_{1}}, a \mathscr{P} \\
i \frac{\mu_{1}}{\lambda_{1}+2 \mu_{1}} a \mathscr{S} & a \mathscr{I}
\end{array}\right), \quad a=\frac{1}{1-\left(\frac{\mu_{1}}{\lambda_{1}+2 \mu_{1}}\right)^{2}}
$$

multipliziert, dann folgt wegen $\mathscr{S}^{2}=\mathscr{I}($ s. $[20:$ S. 41])

$$
\begin{aligned}
\hat{\Phi}_{1} & +\left(a \mathscr{V}_{11}-i \frac{\mu_{1}}{\lambda_{1}+2 \mu_{1}} a \mathscr{S} \mathscr{V}_{21}\right) \hat{\Phi}_{1} \\
& +\left(a \mathscr{V}_{12}-i \frac{\mu_{1}}{\lambda_{1}+2 \mu_{1}} a \mathscr{S} \mathscr{V}_{22}\right) \hat{\Phi}_{2}=a \hat{q}_{1}-i \frac{\mu_{1}}{\lambda_{1}+2 \mu_{1}} a \mathscr{P} \hat{q}_{2} \\
\hat{\Phi}_{2} & +\left(i \frac{\mu_{1}}{\lambda_{1}+2 \mu_{1}} a \mathscr{S} \mathscr{V}_{11}+a \mathscr{V}_{21}\right) \hat{\Phi}_{1} \\
& +\left(i \frac{\mu_{1}}{\lambda_{1}+2 \mu_{1}} a \mathscr{S} \mathscr{V}_{12}+a \mathscr{V}_{22}\right) \hat{\Phi}_{2}=i \frac{\mu_{1}}{\lambda_{1}+2 \mu_{1}} a \mathscr{P} \hat{q}_{1}+a \hat{q}_{2} .
\end{aligned}
$$

Durch Vertauschung der Integrationsreihenfolge (s. [24: S. 104]) folgt

$$
\pi^{2} i \mathscr{P V}_{k j} \hat{\Phi}_{l}=\int_{L} \frac{M_{k j}(t ; \tau)-M_{k j}(\tau, \tau)}{t-\tau} \hat{\Phi}_{l}(\tau) d \tau
$$

mit

$$
M_{k j}(t, \tau)=\int_{\Sigma} \frac{\hat{h}_{k j}(\sigma, \tau)-\hat{h}_{k j}(\sigma, \sigma)}{\sigma-t} d \sigma \in H^{\alpha^{\prime}}(L \times L)
$$

$\dot{(} \alpha^{\prime}<\alpha$, wenn $\alpha \doteq \dot{1}, \alpha^{\prime}=\alpha$, wenn $\left.\alpha<1\right)$.

Zerlegen wir $\hat{q}_{i}=\chi_{1}(t) \hat{q}_{i}(t)+\chi_{2}(t) \hat{q}_{i}(t)$, wobei $\chi_{i}(t)$ genügend glatt ist und $\chi_{i}(t) \geqq \dot{0}$ für $t \in L, \varkappa_{1}(t)=1$ für 't $t \in L \backslash\left(K\left(1, \frac{\varepsilon}{2}\right) \cup K\left(-1, \frac{\varepsilon}{2}\right)\right), \varkappa_{1}(t)=0$ für $t \in L$ $n\left(K\left(1, \frac{\varepsilon}{4}\right) \cup K\left(-1, \frac{\varepsilon}{4}\right)\right), \chi_{2}(t) \doteq 0$ für $t \in L \backslash\left(K\left(1, \frac{\varepsilon}{2}\right) \cup K\left(-1, \frac{\varepsilon}{2}\right)\right), \chi_{2}(\ell)$ $=1$ für $t \in L \cap\left(K\left(1, \frac{\varepsilon}{4}\right) \cup K\left(-1, \frac{\varepsilon}{4}\right)\right), \psi_{1}(t)+\psi_{2}(t)=1$ für $t \in L$. Dann ist $\varkappa_{1}(t) \hat{q}_{i}(t) \in C^{0, \beta}(L)$ und $\%_{2}(t) \hat{q}_{i}(t)=0$ für $t \in L \backslash\left(K\left(1, \frac{\varepsilon}{2}\right) \cup K\left(-1, \frac{\varepsilon}{2}\right)\right)$. Dar- 
aus folgt $\mathscr{P} \hat{q}_{i} \in L_{2}(L) \cap C^{0, \beta}(L \backslash(K(1, \varepsilon) \cup K(-1, \varepsilon)))$ (s. [24: S. 94]). Also kann man (3.19) schreiben

$$
\hat{\boldsymbol{p}} \stackrel{\dot{-}}{\mathscr{V}} \hat{\boldsymbol{p}}=\boldsymbol{g}
$$

wobei die Kerne $v_{i j}(t, \tau)$ des Matrixintegraloperators $\mathscr{V}$ die Gestalt haben $v_{i j}(t, \tau)$ $=\frac{c_{i j}(t, \tau)-c_{i j}(t, t)}{t-\tau} \in H^{\alpha^{\prime}}(L \times L) \quad$ und $g \in L_{2}{ }^{2}(L) \cap C^{0, \beta}(L \backslash(K(1, \varepsilon) \cup K(-1, \varepsilon)))$ gilt. Beachtet man, daß auch $\mathscr{V} g \in L_{2}{ }^{2}(L) \cap C^{0, \beta}(L \backslash(K(1, \varepsilon) \cap K(-1 ; \varepsilon)))$ gilt, dann folgt in bekannter Weise durch Iteration von (3.20) (s. [24: S. 112-113]) $\hat{\boldsymbol{W}} \in L_{2}{ }^{2}([-1,+1]) \cap C^{0, \beta}([-1+\varepsilon, 1-\varepsilon])$ und damit $\varphi \in L_{2}{ }^{2}(S) \cap C^{0, \beta}\left(S \backslash K_{\varepsilon}\right)$

Wir wenden uns nun dem Nachweis der Fredholm-Eigenschaft des Operators $\mathscr{B}$. (.vgl. (3.5)) zu. 'Dazu setzen wir jetzt voraus $S \subset C_{n}^{1, a}$ (vgl. Def. 1.1). Dann ist $f_{1 i}^{0 *}(t, \tau)=f_{11}^{0-}(t, \tau)=0$,

$f_{12}^{0 \pm}(t, \tau)= \pm K_{3}+\frac{1 \mp t}{(2 \mp t \mp \tau)^{2}} \pm K_{5}+\frac{1 \mp \tau}{(2 \mp t \mp \tau)^{2}} \mp 4 \mu_{1} H_{11}^{+} \frac{(1 \mp t)(1 \mp \tau)}{(2 \mp t-\mp \tau)^{3}}$

$f_{13}^{+}(t, \tau)=f_{13}^{0-}(t, \tau)=0$,

$f_{14}^{0 \pm}(t, \tau)= \pm K_{13} \frac{1}{2 \mp t \mp \tau} \mp 2 \mu_{1} H_{4}^{-} \frac{1 \mp t}{(2 \mp t \mp \tau)^{2}} \pm 2 \mu_{1} H_{5}-\frac{1 \mp \tau}{(2 \mp t \mp \tau)^{2}}$,

$f_{21}^{0 \pm}(t, \tau)= \pm K_{2}+\frac{1 \mp t}{(2 \mp t \mp \tau)^{2}} \pm{K_{4}}^{+} \frac{: 1 \mp \tau}{(2 \mp t \mp \tau)^{2}} \pm 4 \mu_{1} H_{11}^{+} \frac{(1 \mp t)(1 \mp \tau)}{(2 \mp t \mp \tau)^{3}}$

$f_{22}^{0}(t, \tau)=f_{22}^{0-}(t, \tau)=0$,

$f_{23}^{0 \pm}(t, \tau)= \pm K_{12}^{-} \frac{1}{2 \mp t \mp \tau} \mp 2 \mu_{1} H_{4^{-}} \frac{1 \mp t}{(2 \mp t \mp \tau)^{2}} \pm 2 \mu_{1} H_{5}^{-} \frac{1 \mp \tau}{(2 \mp t \mp \tau)^{2}}$,

$f_{24}^{0}(t, \tau)=f_{24}^{0-}(t, \tau)=0$,

$f_{31}^{0+}(t, \tau)=f_{31}^{0-}(t, \tau)=0$,

$f_{32}^{0+}(t, \tau)=\mp K_{13}^{+} \frac{1}{2 \mp t \mp \tau} \pm 2 \mu_{0} H_{4}{ }^{+} \frac{1 \mp t}{(2 \mp t \mp \tau)^{2}} \mp 2 \mu_{0} H_{5}{ }^{+} \cdot \frac{1 \mp \tau_{1}}{(2 \mp t \mp \tau)^{2}}$,

$f_{33}^{0+r}(t, \tau)=f_{33}^{0-}(t, \tau)=0$,

$f_{34}^{0 \pm}(t, \tau)=\mp K_{3}^{-} \frac{1 \mp t}{(2 \mp t \mp \tau)^{2}} \mp K_{5}{ }^{-} \frac{1 \mp \tau}{(2 \mp t \mp \tau)^{2}} \pm 4 \mu_{0} H_{11}^{-} \frac{(1 \mp t)(1 \mp \tau)}{(2 \mp t \mp \tau)^{3}}$,

$f_{41}^{0 \pm}(t, \tau)=\mp K_{12}^{\prime} \frac{1}{2 \mp t \mp \tau} \pm 2 \mu_{0} H_{4}+\frac{1 \mp t}{(2 \mp t \mp \tau)^{2}} \mp 2 \mu_{0} H_{5}+\frac{1 \mp \tau^{\prime}}{(2 \mp t \mp \tau)^{2}}$,

$f_{42}^{0+}(t, \tau)=f_{42}^{0-}(t, \tau)=0$,

$f_{43}^{0 \pm}(t, \tau)=\mp K_{2}^{-} \frac{\cdot 1 \mp t}{(2 \mp t \mp \tau)^{2}} \mp K_{4}^{-} \cdot \frac{1 \mp \tau}{(2 \mp t \mp \tau)^{2}} \mp 4 \mu_{0} H_{11}^{-} \frac{(1 \mp t)-(1 \mp \tau)}{(2 \mp t \mp \tau)^{3}}$,

$f_{44}^{0+}(t, \tau)=f_{44}^{0-}(t, \tau)=0$.

Der Operator $\mathscr{B}$ ist also von der Gestalt

$$
\mathscr{B} \Phi=\Phi(t)+\frac{1}{\pi} \int_{-1}^{+1} k^{0}(\ell, \tau) \Phi(\tau) d \tau+\mathscr{X} \Phi
$$


Dabei ist $\mathscr{K}$ ein vollstetiger. Operator von $L_{2}^{4}([-1,+1])$ in $L_{2}{ }^{4}([-1,+1])$ und

$$
\begin{aligned}
k_{i j}^{0}(t, \tau)= & \alpha_{i j} \frac{1}{t-\tau}+\beta_{i j} \frac{1}{2-t-\tau}-\beta_{i j} \frac{1}{2+t+\tau} \\
& +\gamma_{i j} \frac{1-t}{(2-t-\tau)^{2}}-\gamma_{i j} \frac{1+t}{(2+t+\tau)^{2}} \\
& +\eta_{i j} \frac{(1-t)^{2}}{(2-t-\tau)^{3}}-\eta_{i j} \frac{(1+t)^{2}}{(2+t+\tau)^{3}}
\end{aligned}
$$

mit $\alpha_{i j}$ nach (3.6),

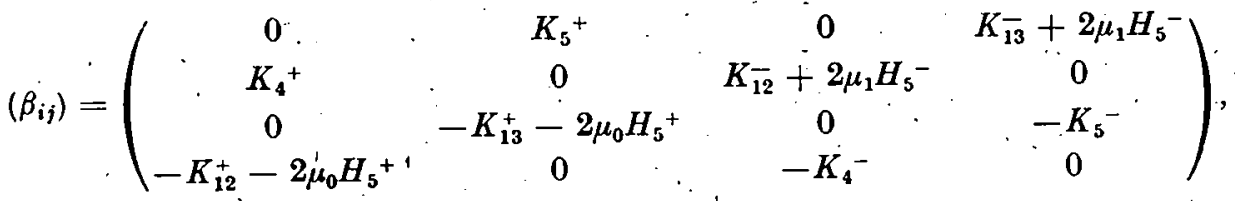

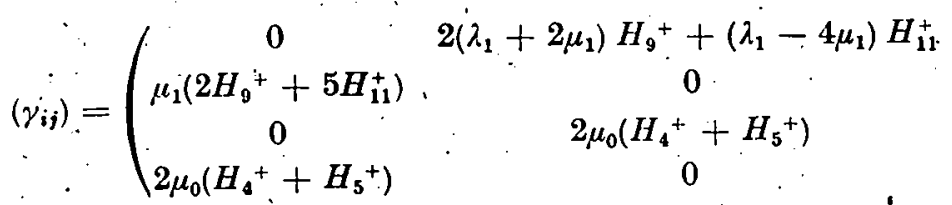

$$
\begin{aligned}
& \left.\begin{array}{ccc}
-2 \mu_{1}\left(H_{4}^{-}+H_{5}^{-}\right) & -2 \mu_{1}\left(H_{4}^{-}+H_{5}^{-}\right) \\
0 & \therefore-2\left(\lambda_{0}+2 \mu_{0}\right) H_{9}^{-}-\left(\lambda_{0}-4 \mu_{0}\right) H_{11}^{-}
\end{array}\right) \\
& \left(\eta_{i j}\right)=\left(\begin{array}{cccc}
0 & 4 \mu_{1} H_{11}^{+} & 0 & 0 \\
-4 \mu_{1} H_{11}^{+} & 0 & 0 & 0 \\
0 & 0 & 0 & -4 \mu_{0} H_{11}^{-} \\
0 & 0 & 4 \mu_{0} H_{11} & 0
\end{array}\right)
\end{aligned}
$$

Die Sýmbole des Matrixoperators $\mathscr{B}$ als Operator von $L_{2}^{4}([-1,+1])$ in $L_{2}{ }^{4}([-1,+1])$ sind (s. [3: (10.4)]):

$$
A_{k j}(\lambda, \xi)=\left\{\begin{array}{l}
\delta_{k j}-i \alpha_{k j} \tanh \pi \lambda-\frac{1}{\cosh \pi \lambda}\left[\beta_{k j}+\gamma_{k j}\left(\frac{1}{2}-i \lambda\right)\right. \\
\left.+\frac{1}{2} \eta_{k j}\left(\frac{3}{2}-i \lambda\right)\left(\frac{1}{2}-i \lambda\right)\right] \text { für }-\infty<\lambda \leq+\infty, \xi=-\infty, \\
\delta_{k j}+i \alpha_{k j} \tanh \pi \xi+\frac{1}{\cosh \pi \xi}\left[\beta_{k j}+\gamma_{k j}\left(\frac{1}{2}-i \xi\right)\right. \\
\left.+\frac{1}{2} \eta_{k j}\left(\frac{3}{2}-i \xi\right)\left(\frac{1}{2}-i \xi\right)\right] \text { für } \lambda=\infty,-\infty<\xi \leqq+\infty
\end{array} .\right.
$$


Wegen

$$
\begin{aligned}
& \lim _{\lambda \rightarrow-\infty} A_{k j}^{\prime}(\lambda,-\infty)=\delta_{k j}+i \alpha_{k j}=A_{k j}(\infty, \infty) \text { und } \\
& \lim _{\xi \rightarrow-\infty} A_{k j}(\infty, \xi)=\delta_{k j}-i \alpha_{k j}=\dot{A}_{k j}(\infty,-\infty) .
\end{aligned}
$$

bildet die Menge der Punkte $A_{k j}(\lambda, \xi)$ in der komplexen Ebene eine geschlossene Kurve, die im Sinne wachsender $\lambda, \xi$ orientiert sei. Ebenso ist dann $\operatorname{det}\left(A_{k j}(\lambda, \xi)\right)$. eine geschlossene orientierte Kurve. Wenn $\inf \left|\operatorname{det}\left(A_{k j}(\lambda, \xi \xi)\right)\right|>0$ ist, dann sei ind $\left(A_{k j}\right)$ gleich der Anzahl der mathematisch positiven minus der Anzahl der mathematisch negativen Umfahrungen der Kurve $\operatorname{det}\left(A_{k j}(\lambda, \xi)\right)$ um die komplexe Zahl $z=0$.

Es. gilt nun (s. [3: Satz 12.5, Satz 12.6]), der folgende Fakt.

Satz 3.4: Es ist $\mathscr{B}$ in $\dot{L}_{2}^{4}([-1,+1])$ dann und nur dann ein Noetheroperator, wenn

$$
\underset{\lambda, \xi}{\inf }\left|\operatorname{det}\left(A_{k j}(\lambda, \xi)\right)\right|>0 \text {. }
$$

Ist (3.23) erfüllt, dann gilt für den Index von $\mathscr{B}$

Ind $\mathscr{B} .=-$ ind $\left(A_{k j}\right)$.

Setzen wir $f(\lambda, \xi)=\operatorname{det}\left(A_{k j}(\lambda, \xi)\right)$, dann zeigt man leicht

$$
f(\lambda,-\infty)=f(\infty, \lambda)
$$

Daraus 'folgt, daß $f(\lambda):=f(\lambda,-\infty),-\infty<\lambda \leqq+\infty$, eine geschlossene Kurve ist, die wir kurz Symbolkurve nennen: Weiter folgt aus (3.24), daß die Kurve $f(\lambda, \xi)$ gleich der zweimal mit derselben Orientierung durchfahrenen Symbolkurve $f(\lambda)$ ist. Man stellt -leicht fest, daß Re $f\left(\lambda_{0}\right)$ eine gerade und $\operatorname{Im} f(\lambda)$ eine ungerade Funktion ist. Also ist die Symbolkurve $f(\lambda)$ symmetrisch zur reellen Achse. Es ist

$$
f(\infty)=\left[1-\left(\frac{\mu_{0}}{\lambda_{0}+2 \mu_{0}}\right)^{2}\right]\left[1-\left(\frac{\mu_{1}}{\lambda_{1}+2 \mu_{1}}\right)^{2}\right]
$$

Unter den in der Elastizitätstheorie üblichen Voraussetzungen $\lambda_{i}>0, \mu_{i}>0$ gilt $\frac{9}{16}<f(\infty)<1$. Aus diesen Utberlegungen folgt mit Satz 3.4 eine weitere Aussage.

Satz 3.5: Es sei $S \subset C_{n}^{1, \alpha}(0<\alpha \leqq 1)$ und

$$
\operatorname{Re} f(\lambda)>0 \text { fïr } 0 \leqq \lambda<\infty \text {. }
$$

Dann ist $\mathscr{B}$ in $L_{2}^{4}([-1,+1])$ ein Noetheroperator mit Ind $\mathscr{B}=0$.

Wenn nun $\hat{\lambda}_{0}=\lambda_{1} \doteq \lambda^{*}, \mu_{0}=\mu_{1}=\mu^{*}$ ist, dann ist

$$
\begin{aligned}
& H_{1}^{-}=H_{1}^{+}=\frac{\lambda^{*}+3 \mu^{*}}{2 \mu^{*}\left(\lambda^{*}+2 \mu^{*}\right)}, H_{2}{ }^{-}=H_{2}{ }^{+}=-\frac{\lambda^{*}+\mu^{*}}{2 \mu^{*}\left(\lambda^{*}+2 \mu^{*}\right)} \\
& H_{3}{ }^{-}=H_{3}{ }^{+}=0, \\
& H_{4}^{-}=H_{4}^{+}=-\frac{\lambda^{*}+\mu^{*}}{2 \mu^{*}\left(\lambda^{*}+2 \mu^{*}\right)}, \quad H_{5}{ }^{-}=H_{5}{ }^{+}=\frac{\lambda^{*}+\mu^{*}}{2 \mu^{*}\left(\lambda^{*}+2 \mu^{*}\right)}, \\
& H_{k^{-}}=H_{k^{+}}{ }^{+}=0 \text { für } k=6, \ldots, 11
\end{aligned}
$$


und man erhält

$$
f(\lambda)=\left[1-\left(\frac{\mu^{*}}{\lambda^{*}+2 \mu^{*}}\right)^{2}\right]^{2},
$$

d. h., díe Symbolkurve zieht sich auf einen Punkt zusammen, der auf der reellen Achse im Intervall $\left(\frac{9}{16}, 1\right)$ liegt. Da man den Fall allgemeiner Laméscher Moduln durch stetige Deformation aus dem Fall gleicher Laméscher Moduln erzeugen kann, ist (3.26) sicher erfüllt, wenn sich $\mu_{0}$ von $\mu_{1}$ und $\lambda_{0}$ von $\lambda_{1}$ nicht zu viel unterscheiden.

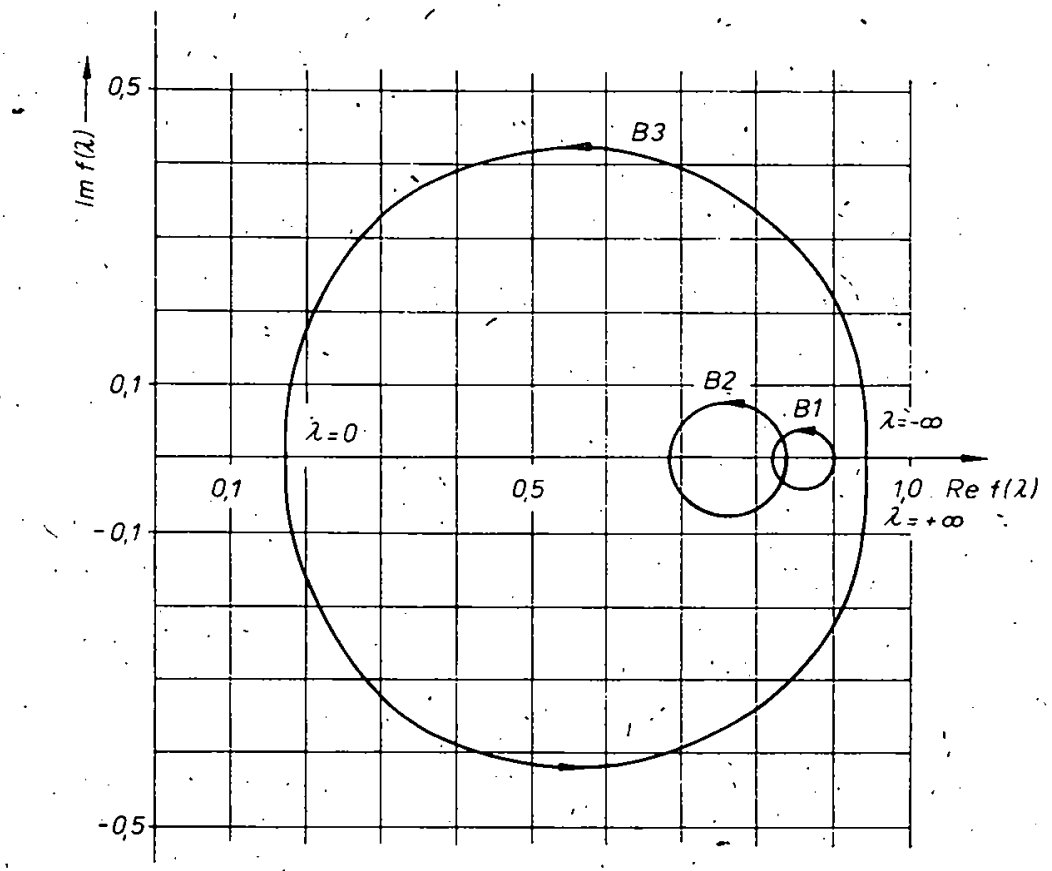

Abb. 2

In den drei folgenden gerechneten Beispielen war Bedingung (3.26) erfüllt (s. Abb. 2):

B 1: $\mu_{0}=0,2, \lambda_{0}=0,6 ; \mu_{1}=0,4, \lambda_{1}=0,8$,

B2: $E_{0}=7300, v_{0}=0,25(\mathrm{Al}) ; E_{1}=22000, v_{1}=0,33(\mathrm{Fe})$,

B 3: $E_{0}=0,1, v_{0}=0,4 ; E_{1}=100, v_{1}=0,4$.

Für die Lage des Punktes $f(0)$ im Grenzfall $E_{0} / E_{1}=0\left(E_{1} / E_{0}=0\right.$ liefert dasselbe, da sich $f(\lambda)$ bei Vertauschung der Indizes bei den Laméschen Moduln nicht ändert). ergibt sich

$$
f(0)=\frac{9-24 v_{1}+16 v_{1}^{2}}{16\left(1-v_{1}\right)^{2}} \cdot \frac{17-48 v_{0}+32 v_{0}^{2}}{16\left(1-v_{0}\right)^{2}}=: g\left(v_{0}, v_{1}\right) .
$$

Daraus folgt

$$
\frac{153}{256}=g(0,0) \geqq g\left(v_{0}, \nu_{1}\right) \geqq g\left(\frac{1}{2}, \frac{1}{2}\right)=\frac{1}{16} \quad \text { für } \quad 0 \leqq v_{0}, \bar{v}_{1} \leqq \frac{1}{2} .
$$


Das legt die Vermutung nahe, daß (3.26) unter der Voraussetzung $E_{i}>0,0<v_{i}<\frac{1}{2}$ immer erfüllt ist. Ein allgemeiner Beweis dieser Vermutung ist auf-Grund der Kompliziertheit von $f(\lambda)$ nicht gelungen.

Satz 3.6: Es sei $S \subset C_{n}^{1, \alpha}(0<\alpha \leqq 1)$ und (3.26) erfüllt. Dann ist. $N\left(\dot{A}^{*}\right)$ $\stackrel{\mathscr{L}}{=}\left\{\boldsymbol{a}_{1}, \boldsymbol{a}_{2}, \boldsymbol{a}_{3}\right\}$ und entsprechend $N\left(\mathscr{B}^{*}\right)=\mathscr{L}\left\{\Psi_{(1)}^{*}, \Psi_{(2)}^{*}, \Psi_{(3)}^{*}\right\}$.

Beweis: Nach Satz 3.2 ist $\operatorname{dim} N\left(\mathscr{A}^{*}\right) \geqq 3$. Sei $\operatorname{dim} N\left(\mathscr{A}^{*}\right)>3$, dann wäre wegen Ind $\mathscr{B}=$ Ind $\dot{A}=0$ auch $\operatorname{dim} N(\mathscr{A})>3$, d. h. in $N(\mathscr{A})$ gäbe es wenigstens 4 linear unabhängige Vektoren $\varphi_{1}, \ldots, \varphi_{4}$. Aus Satz 3.3 und Satz 2.1 und $\mathscr{A}_{\varphi_{i}}=0$ folgt, da $\beta V\left(x, \dot{\varphi}_{j}\right)$ Lösung des Problems II $(D ; 0,0 ; 0 ; 0,0)$ ist. Aus dem Eindeutigkeitssatz folgt $\boldsymbol{V}\left(x, \varphi_{j}\right) \in \mathscr{L}\left\{\boldsymbol{a}_{1}, \boldsymbol{a}_{1}, \boldsymbol{a}_{3}\right\}$. Dann gibt es in $\mathscr{L}\left\{\varphi_{1}, \ldots, \varphi_{4}\right\}$ (s. [18: S. 33]) einen Vektor $\varphi \neq \mathbf{0}$ mit

$$
\therefore \int_{s} \varphi d s=0, \quad V(x, \varphi)=\sum_{i=1}^{2} \delta_{i} a_{i}(x)=\delta_{1} i_{1}+\delta_{2} i_{2}, \quad x \in D .
$$

Wegen Satz 2.3 ist $V(x, \varphi)$ in $C D$ Lösung des Problems $I\left(C D ; 0,0 ; \delta_{1} i_{1}+\delta_{2} i_{2} ; 0 ; 0\right)$. Nun ist offenbar auch $\delta_{1} i_{1}+\delta_{2} i_{2}$ Lösung dieses Problems, also gilt auf Grund des Eindentigkeitssatzes $V(x, \varphi)=\delta_{1} i_{1}+\delta_{2} i_{2}$ für $\dot{x} \in C D$. Wegen Satz 2.2 gilt $V(x, \varphi)$ $=o(1)$, also ist $\delta_{1}=0, \delta_{2}=0$ und somit $\dot{V}(x, \varphi) \equiv 0$. Aus der Sprungrelation für das elastische' Potential der einfachen Schicht (s. [18: (4.2), (4.3)]) folgt $\varphi \doteq 0$. Dieser Widerspruch zeigt, $\operatorname{da} \beta \operatorname{dim} N\left(\mathscr{A}^{*}\right)=3$ ist und somit $N\left(\mathscr{A}^{*}\right)=\mathscr{L}\left\{\boldsymbol{a}_{1}, \boldsymbol{a}_{2}, \boldsymbol{a}_{3}\right\}$ ist

\section{Existenzsätze}

Sat, 4.1: Es sei $S \subset C_{n}^{1, \alpha}$, Bedingung (3.26) erfüllt, $\boldsymbol{P} \in L_{2}{ }^{2}(S) \cap C^{0, \beta}\left(S \backslash K_{\varepsilon}\right)$ für jedes $\varepsilon>0,0<\beta<\alpha \leqq$ 1. Das Problem $\mathrm{II}(D ; 0,0 ; P ; 0,0)$ besitzt dann und nur dann eine Lösung $\boldsymbol{u}(x)$, wenn die Gleichgèwichtsbedingungen

$$
\int_{s} \boldsymbol{P} \cdot \boldsymbol{a}_{k} d s=0, \quad k=1,2,3
$$

erfüllt sind. Es ist $\boldsymbol{u}(x)$ darstellbar als Potential $\boldsymbol{u}(x)=\boldsymbol{V}(x, \varphi)$, wobei $\boldsymbol{\rho} \in L_{\mathbf{2}}{ }^{2}(S)$ $\cap C^{0 . \beta}\left(S \backslash K_{\varepsilon}\right)$ eine Lösung vón $\mathscr{A} \varphi=P$ ist.

Beweis: Daß (4.1) notwendig ist, haben wir bereits festgestellt (s. (1.8)). Sei nun (4.1) erfiillt. Dann gilt

$$
\begin{aligned}
\int_{-1}^{+1} \Psi_{(k)}^{*}(t) \cdot p(t) d t= & \int_{-1}^{+1} \boldsymbol{a}_{k}\left(y^{+}(t)\right)\left|\dot{y}^{+}(t)\right| \cdot \boldsymbol{P}\left(y^{+}(t)\right) d t \\
& +\int_{-1}^{+1} \boldsymbol{a}_{k}\left(y^{-}(t)\right)\left|\dot{y}^{-}(t)\right| \cdot \boldsymbol{P}\left(y^{-}(t)\right) d t \\
= & \int_{s^{+}} \boldsymbol{a}_{k} \cdot \boldsymbol{P} d s+\int_{s^{-}} \boldsymbol{a}_{k} \cdot \boldsymbol{P} d s=\int_{s} \boldsymbol{a}_{k} \cdot \boldsymbol{P} d s=0
\end{aligned}
$$

Also hat $\mathscr{B} \Phi=p$ und damit $\mathscr{A} \varphi=P$ eine Lösung. Aus Satz 3.3, Satz 2.4, $\mathscr{A} \varphi=P$ folgt, daß $u(x)=V(x, \varphi)$ Lösung des Problems II $(D ; 0,0 ; P ; 0,0)$ ist

Wir kommen jetzt zum allgemeinen Fall:

Wir betrachten ein Kurvenstiick $S_{1}$ der oberen Halbebene, so daß $S_{0}^{*}=S_{0} \cup \bar{S}_{1}$ , eine beliebig glatte geschlossene Kurve ist und das Innengebiet $D^{+*}$ von $S_{0}^{*}$ das 
Gebiet $D^{+}$enthält. Sei $D^{+*} \cup D \subset K(0, R)$ und $D^{-*}=K(0, R) \backslash \overline{D^{+*}}, S_{R}=|x:| x \mid$ $=R\}$. Wir betrachten folgendes Rand-Kontaktproblem $K^{*}\left(0,0 ; \boldsymbol{w}^{*}, \boldsymbol{P}^{*}\right)$ :

$$
\begin{aligned}
& A \boldsymbol{u}=\mathbf{0} \text { in } D^{+*}, \quad \underset{(0)}{A \boldsymbol{u}=\mathbf{0}} \text { in } D^{-*}, \\
& \{\boldsymbol{u}\}^{+}-\{\boldsymbol{u}\}^{-}=\boldsymbol{w}^{*} \quad \text { auf } S_{0}^{*},\left\{\begin{array}{l}
T\left(\partial_{x}, \boldsymbol{n}\right) \boldsymbol{u} \\
(1)
\end{array}\right\}^{+}-\left\{\begin{array}{l}
\left.T\left(\partial_{x}, \boldsymbol{n}\right) \boldsymbol{u}\right\}^{-}=\boldsymbol{P}^{*} \quad \text { auf } \\
S_{0^{*}}, \boldsymbol{u}=\mathbf{0} \text { auf } \quad S_{R} .
\end{array}\right.
\end{aligned}
$$

Es gilt (s. [18], vgl. auch [5]) folgende Aussage.

Hilfssatz 4.1: Unter den Voraussetzungen w* $w^{*} C^{1,8}\left(S_{0}^{*}\right), P^{*} \in C^{0,8}\left(S_{0}^{*}\right), 0<\beta$ .$<1$, hat das Problem $K^{*}\left(0,0 ; \boldsymbol{w}^{*}, \boldsymbol{P}^{*}\right)$ eine eindeutige Lösung $\boldsymbol{u}$, für die gilt:

$$
\left.\boldsymbol{u}\right|_{D^{+*}} \in \dot{C}^{1, \beta}\left(D^{+*}\right) \cap C^{\infty}\left(D^{+*}\right),\left.\quad \boldsymbol{u}\right|_{D^{-*}} \in C^{1, \beta}\left(D^{-*}\right) \cap C^{\infty}\left(D^{-*}\right) .
$$

Gegeben seien die Kontaktdaten $\hat{w} \in C^{1, \beta}\left(S_{0}\right), \hat{\boldsymbol{P}} \in C^{0, \beta}\left(S_{0}\right)$, die , Volumenkraft $\left.\boldsymbol{F}\right|_{D^{+}} \in C^{0, \beta}\left(D^{+}\right),\left.\boldsymbol{F}\right|_{D^{-}} \in C^{0, \beta}\left(D^{-}\right)$und das Temperaturfeld $\left.\theta\right|_{D^{+}} \in C^{1, \beta}\left(D^{+}\right),\left.\theta\right|_{D^{-}}$ $\in C^{1, \beta}\left(D^{-}\right)$. Wir setzèn $\hat{\boldsymbol{w}}, \hat{\boldsymbol{P}}$ auf $S_{0}^{*}$ fort zu $\hat{\boldsymbol{w}}^{*} \in C^{1, \beta}\left(S_{0}^{*}\right), \hat{\boldsymbol{p}} \boldsymbol{\epsilon} \in C^{0, \beta}\left(S_{0}^{*}\right)$, setzen $\boldsymbol{F}$ fort $\left.\mathrm{zu} \boldsymbol{F}^{*}\right|_{D^{+*}} \in C^{0, \beta}\left(\dot{D}^{+*}\right),\left.\boldsymbol{F}^{*}\right|_{D^{-*}} \in C^{0, \beta}\left(D^{-*}\right)$ und setzen $\operatorname{grad} \theta$ fort zu grad $\left.\theta^{*}\right|_{D^{+*}}$ $\in C^{0, \beta}\left(D^{+*}\right)$, grad $\left.\theta^{*}\right|_{D^{-*}} \in C^{0.8}\left(D^{-*}\right)$. Auf Grund eines Lemmas in [4: S. 556] braucht man die Fortsetzbarkeit von $\boldsymbol{F}$ (bzw. grad $\theta$ ) nur in der Umgebung jedes Randpunktes $x^{*}$ von $D^{+}$einzusehen. Falls $S \subset C_{n}^{1, \alpha}$, bilden wir $K\left(x^{*}, \varepsilon\right) \cap D^{+}$auf ein Halbebenenstück bzw. ein Quadrantenstück ab. Eine $C^{0 . \beta}$-Funktion der Halbebene kann man offenbar fortsetzen; eine $C^{0 . \beta}$-Funktion $f(x)$ eines Quadrànten $Q$ mit dem Eckpunkt $x_{E}$ kann man z. B. so fortsetzen, daß man für $y$ auf. der äußeren Normalen inm Randpunkt $x_{0}$ von $Q$ setzt $f(y)=f\left(x_{0}\right)$ und für $y$ in $\operatorname{dem} Q$ gegenïberliegendem Quadranten $f(y)=f\left(x_{E}\right)$.

Wir betrachten jetzt das Flächenpotential

$$
\boldsymbol{u}_{\theta, \boldsymbol{F}}(x)=\left\{\begin{array}{l}
\frac{1}{2 \pi} \int_{D^{+*}} \Gamma(x, y)\left(\boldsymbol{F}^{*}-\left(2 \mu_{1}+3 \dot{\dot{\lambda}_{1}}\right) \alpha_{1} \operatorname{grad} \theta^{*}\right) d y \text { für } x \in D^{+*} \\
\frac{1}{2 \pi} \int_{D^{*}} \Gamma(x, y)\left(F^{*}-\left(2 \mu_{0}+3 i_{0}\right) \alpha_{0} \operatorname{grad} \theta^{*}\right) d y \text { für } x \in D^{-*}
\end{array}\right.
$$

Dann gilt (s. [18: Satz 4.5]):

$$
\begin{aligned}
& \left.\boldsymbol{u}_{\theta, \boldsymbol{F}}\right|_{D^{+*}} \in C^{2}\left(D^{+*}\right) \cap C^{1, \beta}\left(D^{+*}\right), \quad u_{\theta, F_{D^{-*}}} \in C^{2}\left(D^{-*}\right) \cap C^{1, \beta}\left(D^{-*}\right), \\
& A \boldsymbol{u}_{0, p}=\left(2 \mu_{1}+3 \lambda_{1}\right) \alpha_{1} \operatorname{grad} \theta^{*}-\boldsymbol{F}^{*} \text { in } D^{+*} \text {, } \\
& \text { (1) } \\
& A \boldsymbol{u}_{\theta, \boldsymbol{F}}=\left(2 \mu_{0}+3 \lambda_{0}\right) \alpha_{0} \operatorname{grad} \theta^{*}-\boldsymbol{F}^{*} \text { in } D^{-*}, \\
& \text { (0) } \\
& \left\{\boldsymbol{u}_{\theta, \boldsymbol{F}}\right\}^{+}-\left\{\boldsymbol{u}_{\theta, \boldsymbol{F}}\right\}^{-}=: \widetilde{\boldsymbol{w}} \in C^{1, \beta}\left(S_{0}^{*}\right)
\end{aligned}
$$

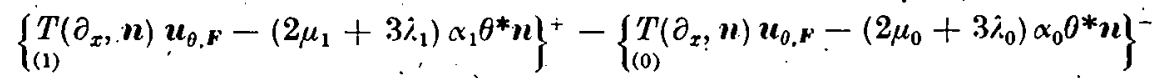

$$
\begin{aligned}
& =: \tilde{\boldsymbol{P}} \in C^{0, \beta}\left(S_{0}^{*}\right) \text {. }
\end{aligned}
$$

Sei jetzt $\boldsymbol{u}^{*}$ die Lösung des Problems $K^{*}\left(\mathbf{0}, 0 ; \dot{\hat{\boldsymbol{w}}}^{*}-\tilde{\boldsymbol{w}}, \hat{\boldsymbol{P}} \boldsymbol{-}-\tilde{\boldsymbol{P}}\right)$, dann hat $\boldsymbol{u}_{p}$ $=\boldsymbol{u}^{*}+\boldsymbol{u}_{\theta, \boldsymbol{F}}$ folgende Eigenschaften:

$$
\left.u_{p}\right|_{D^{+*}} \in C^{2}\left(D^{+*}\right) \cap C^{1, \beta}\left(D^{+*}\right) ;\left.\quad u_{p}\right|_{D^{-*}} \in C^{2}\left(D^{-*}\right) \cap C^{1, \beta}\left(D^{-*}\right),
$$




$$
\begin{aligned}
& A u_{p}=\left(2 \mu_{1}+3 \lambda_{1}\right) \alpha_{1} \operatorname{grad} \theta^{*}-F^{*} \text { für } x \in D^{+*} \text {, } \\
& \text { (1) } \\
& \underset{(0)}{A u_{p}}=\left(2 \dot{\mu_{0}}+3 \lambda_{0}\right) \alpha_{0} \operatorname{grad} 0^{*}-F^{*} \text { für } x \in D^{-*} \text {, } \\
& \left\{\boldsymbol{u}_{p}\right\}^{+}-\left\{\boldsymbol{u}_{p}\right\}^{-}=\hat{\boldsymbol{w}}^{*}
\end{aligned}
$$

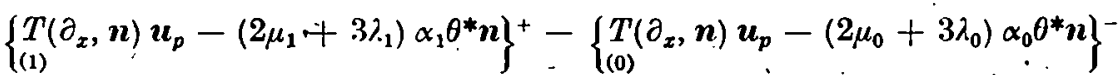

$$
\begin{aligned}
& =\hat{P}^{*} \text {. }
\end{aligned}
$$

Sei nun $\boldsymbol{P} \in L_{2}^{2}(S) \cap C^{0, \beta}\left(S \backslash K_{\varepsilon}\right)$, dánn, gilt auch für

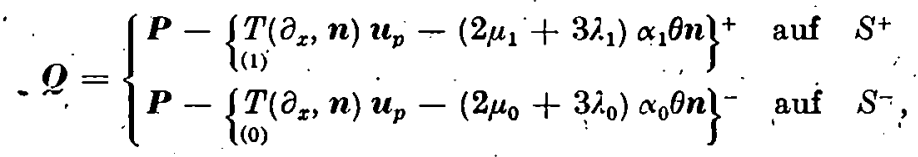

daß $Q \in L_{2}{ }^{2}(S) \cap C^{0 . \beta}\left(S \backslash K_{e}\right)$ ist.

Wendet man diè Bettische Formel (s. [18: (3.13)) an auf $D^{+}, D^{-}$mit $\boldsymbol{u}=\boldsymbol{u}_{\boldsymbol{p}}, \boldsymbol{v}=\boldsymbol{a}_{\mathrm{k}}$, dann folgt

$$
\int_{S} \boldsymbol{Q} \cdot \boldsymbol{a}_{k} d s=\int_{S} \boldsymbol{F} \cdot \boldsymbol{a}_{k} d x_{r}+\int_{S} \boldsymbol{P} \cdot \boldsymbol{a}_{k} d s+\int_{s_{0}} \boldsymbol{P} \cdot \boldsymbol{a}_{k} d s
$$

Setzen wir also die Gleichgewichtsbedingungen (1.8) voraus, dann hat das Problem $\mathrm{II}(D ; 0,0 ; \boldsymbol{Q} ; 0,0)$ eine Lösung $\boldsymbol{u}_{H}$. Es ist dann $\boldsymbol{u}=\boldsymbol{u}_{H}+\left.\boldsymbol{u}_{p}\right|_{\boldsymbol{D}}$ eine Lösung des Problems II $(D ; \boldsymbol{F}, \theta ; \boldsymbol{P} ; \hat{\boldsymbol{w}}, \hat{\boldsymbol{P}})$. Damit haben wir den folgenden Fakt.

Satz 4.2: Sei $S \subset C_{n}{ }^{1, \alpha}, B$ Bedingung (3.26) erfüllt, $\boldsymbol{P} \in L_{2}{ }^{2}(S)$ in $C^{0 . \beta}\left(S \backslash K_{\varepsilon}\right)$ für jedes $\varepsilon>0, \quad 0<\beta<\alpha \leqq 1, \quad \hat{\boldsymbol{w}} \in C^{1, \beta}\left(S_{0}\right), \quad \hat{\boldsymbol{P}} \in C^{0, \beta}\left(S_{0}\right),\left.\quad \boldsymbol{F}\right|_{D^{+}} \in C^{0, \beta}\left(D^{+}\right)$, $\left.\boldsymbol{F}\right|_{D^{-}} \in C^{0, \beta}\left(D^{-}\right),\left.0\right|_{D^{+}} \in C^{1, \beta}\left(D^{+}\right),\left.0\right|_{D^{-}} \in C^{1, \beta}\left(D^{-}\right)$. Das Problem $\operatorname{II}(D ; \mathrm{F}, 0 ; \boldsymbol{P} ; \hat{\boldsymbol{w}}, \hat{\boldsymbol{P}})$ besitžt dann und nur dainn eine Lösung, wenn die Gleichgewichtsbedingungen (1.8) erfüllt sind.

Zum Schluß geben wir noch eine explizite partikuläre Lösung an.

Satz 4.3: Sei $S \subset C_{n}{ }^{i, \alpha}, \dot{\boldsymbol{w}}, \hat{\boldsymbol{P}}, \boldsymbol{F}, \theta$ erfülle die Voraussetzungen des Satzes 4.2, und es sei $\hat{\boldsymbol{w}}\left(y^{+}( \pm 1)\right)=\mathbf{0}$. Dann ist

$$
\begin{aligned}
& \boldsymbol{u}_{G}(x)=\frac{1}{2 \pi} \int_{D^{+}}{ }^{\prime}\left(\mathrm{i}(x, y)\left[\boldsymbol{F}-\left(2 \mu_{1}+3 \dot{\lambda}_{1}\right) \alpha_{1} \operatorname{grad} \theta\right] d y\right. \\
& +\frac{1}{2 \pi} \int_{D^{-}} \mathbf{G}(x, y)\left[\boldsymbol{F}-\left(2 \mu_{0}+3 \hat{\lambda}_{0}\right) \alpha_{0} \operatorname{grad} \theta\right] d y, \\
& +\frac{1}{2 \pi} \int_{S_{0}} \mathbf{G}(x, y)\left[\hat{\boldsymbol{P}}+\left(2 \mu_{1}+3 \dot{\lambda}_{1}\right) \alpha_{1}\{\theta\}^{+} \cdot \boldsymbol{n}\right. \\
& \left.-\left(2 \mu_{0}+3 \lambda_{0}\right) \alpha_{0}\{0\}-n\right] d_{\nu} s
\end{aligned}
$$

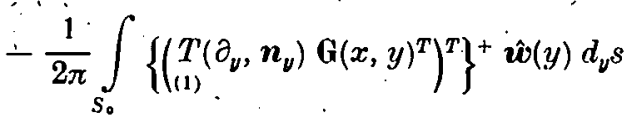

eine Lösung des Problems $\mathrm{I}\left(D ; \boldsymbol{F}, \theta ;\left\{\boldsymbol{u}_{G}\right\}, \hat{\boldsymbol{w}}, \hat{\boldsymbol{P}}\right)$. Für den Grenzwert $\left\{T \boldsymbol{u}_{G}\left(x_{0}\right)\right\}(s$. Satz 2.5) gilt $\left\{T u_{G}\left(x_{0}\right)\right\} \in L_{2}{ }^{2}(S) \cap C^{0, \beta}\left(S \backslash K_{e}\right)$. 
Beweis: Für die oben konstruierte partikuläre Lösung $\boldsymbol{u}_{p}$ erhält mán mit Hilfe der Bettischen Formel die Darstellung

$$
\boldsymbol{u}_{p}(\dot{x})=\boldsymbol{u}_{G}(x)+\boldsymbol{u}_{H_{1}}(x)-\boldsymbol{u}_{H_{3}}(x) \text { für } x \in D^{+} \cup \dot{D^{-}}
$$

Dabei ist

$$
\boldsymbol{u}_{H_{1}}(x)=\frac{1}{2 \pi} \int_{s^{\prime}} \mathbf{G}(x, y), y,(y) d_{\nu} s
$$

mit

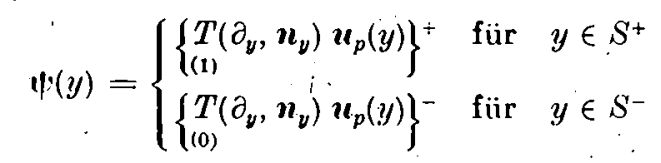

und $\boldsymbol{u}_{H}$, jst in Satz 2.5 definiert. Aus Satz 2.4 und Satz 2.5 und den Eigenschaften von $\boldsymbol{u}_{p}$ folgt, da. $\beta \boldsymbol{u}_{G}(x)$ Lösung des Problems $\mathrm{I}\left(D ; \boldsymbol{F}, \theta ;\left\{\boldsymbol{u}_{G}\right\} ; \boldsymbol{\boldsymbol { w }}, \hat{\boldsymbol{P}}\right)$ ist. Offenbar ist $\left\{T u_{p}\left(x_{0}\right)\right\} \in L_{2}^{2}(S) \cap C^{0, \beta}\left(S \backslash K_{\varepsilon}\right)$, und es ist $\left\{T u_{H}\left(x_{0}\right)\right\} \in L_{2}^{2}(S) \cap C^{0, \beta}\left(S \backslash K_{\varepsilon}\right)$ auf Grund von Satz 2.5. Da $\psi \in L_{2}{ }^{2}(S)$ und $\left\{T u_{H_{1}}\left(x_{0}\right)\right\}=\mathscr{A} \psi$ gilt und $\mathscr{A}$ ein linearer beschränkter Operator von $L_{2}{ }^{2}(S)$ in $L_{2}{ }^{2}(S)$ ist, folgt auch $\left\{T u_{H_{1}}\left(x_{0}\right)\right\} \in L_{2}{ }^{2}(S)$. Damit ist der Satz bewiesen

Auf Grund von Satz 4.3 läßt sich ène Iösung des Problems $\operatorname{II}(D ; \boldsymbol{F}, 0 ; \boldsymbol{P} ; \dot{\hat{\boldsymbol{w}}}, \hat{\boldsymbol{P}})$ darstellen als Summe von $\boldsymbol{u}_{G}(x)$ und einem Potential $\boldsymbol{V}(x, \boldsymbol{\rho})$ (vgl. [12:(19)]).

\section{Anhang}

Die Konstanten $H_{\boldsymbol{k}}$ - sind :

$$
\begin{aligned}
& H_{1}^{-}=4\left(1+v_{0}\right)\left(1+v_{1}\right) \\
& \times \frac{E_{1}\left(1+v_{0}\right)\left(3-4 v_{0}\right)\left(1-\nu_{1}\right)+E_{0}\left(1-v_{0}\right)\left(1-v_{1}\right)\left(3-4 v_{1}\right)}{\left[E_{0}\left(3-4 \nu_{1}\right)\left(1+v_{1}\right)+E_{1}\left(1+v_{0}\right)\right]\left[E_{1}\left(3-4 v_{0}\right)\left(1+v_{0}\right)+E_{0}\left(1+v_{1}\right)\right]}, \\
& H_{2}^{-}=H_{1}^{-}-\frac{4\left(1+v_{0}\right)\left(1+v_{1}\right)}{E_{0}\left(1+v_{1}\right)+^{\prime} E_{1}\left(1+v_{0}\right)}, \\
& H_{3}^{-} \doteq 2\left(1+v_{0}\right)\left(i+v_{1}\right) \\
& \times \frac{E_{0}\left(1-2 v_{0}\right)\left(1+v_{1}\right)\left(3-4 v_{1}\right)-E_{1}\left(1+v_{0}\right)\left(3-4 v_{0}\right)\left(1-2 v_{1}\right)}{\left[E_{0}\left(3-4 v_{1}\right)\left(1+v_{1}\right)+E_{1}\left(1+v_{0}\right)\right]\left[E_{1}\left(3-4 v_{0}\right)\left(1+v_{0}\right)+E_{0}\left(1+v_{1}\right)\right]}, \\
& H_{4}^{-}=-\frac{2\left(1+v_{0}\right)\left(1+\nu_{1}\right)}{E_{0}\left(3-4 v_{1}\right)\left(1+\nu_{1}\right)+E_{1}\left(1+\nu_{0}\right)} \\
& H_{5}^{-}=\frac{2\left(1+\nu_{0}\right)\left(1+\nu_{1}\right)}{E_{3}\left(3-4 \nu_{0}\right)\left(1+\nu_{0}\right)+E_{0}\left(1+\nu_{1}\right)} \\
& H_{6}^{-}=H_{1}^{-}-\frac{\left(3 \div 4 \nu_{0}\right)\left(1+\nu_{0}\right)}{2 E_{0}\left(1-v_{0}\right)}, \quad H_{-}^{-}=H_{2}^{-}+\frac{1+v_{0}}{2 E_{0}\left(1-v_{0}\right)}, \quad H_{8}^{-}=H_{3}^{-} \text {， }
\end{aligned}
$$


$H_{9}^{-}=\frac{1}{2} \frac{\left(1+v_{0}\right)\left(3-4 v_{0}\right)\left[E_{0}\left(1+v_{1}\right)-E_{1}\left(1+v_{0}\right)\right]}{\left(1-v_{0}\right) E_{0}\left[E_{1}\left(3-4 v_{0}\right)\left(1+v_{0}\right)+E_{0}\left(1+v_{1}\right)\right]}, \quad H_{10}^{-}=H_{9}^{-}$,

$H_{11}^{-}=\frac{\left(1+\nu_{0}\right)\left[E_{1}\left(1+v_{0}\right)-E_{0}\left(1+v_{1}\right)\right]}{\left(1-v_{0}\right) E_{0}\left[E_{1}\left(3-4 v_{0}\right)\left(1+\nu_{0}\right)+E_{0}\left(1+\nu_{1}\right)\right]}$.

Die Konstanten $\dot{H}_{k}{ }^{+}$ergeben sich aus $\dot{H}_{k}{ }^{-}$durch Vertauschung der Indizes $\dot{0}$ und 1.

\section{LITERATUR}

[1] Beckért, H.: Utber die klassischen Randwertaufgaben in der Theorie der Wärmespannungen in stückweise stetigen, anisotropen Körpern, unter Kopplungsbedingungen. ZAMM 52 (1972), $111-122$.

[2] Dempsey, J. P., and G. B. Srnclatr: On the stress singularities in the plane elasticity of the composite wedge. J. Elasticity $9(1979)$, no. 4, 373-391.

[3] Dondchava, R.: Integral equations with fixed singularities. Teubner-Texte zur Mathematik. Leipzig 1979.

[4] Ficutenuolz, G. M.: Differential- und Integralrechnung I. VEB Deutscher Verlag der Wissenschaften: Berlin 1969.

[j] Jestsch, L.: Uber Wärmespannungen in Körpern mit stückweise konstanten La méschen Elastizitätsmoduln. Schriftenreihe des ZIMM bei der Akademie der. Wissenschaften der DDR, Heft 14. Akademie-Verlag: Berlin 1972.

[6] JEsTSCH, L. : Zur Existenz von regulären Lösungen der Elastostatik stückwcise homogener Körper mit neuen Kontaktbedingungen an den Trennflächen zwischen zwei homogenen Teilen. Abhandlungen der Sächs. Akademie der Wissensch. zu Leipzig, Math.-naturiw. Klasse Bd. 53, Heft 2, 1977.

[7] Jestsch, L.: Bemerkungen zu einigen neueren gekoppelten Randwertproblemen der Thermoelastostatik. Wiss. Z. Karl-Marx-Univ. Leipzig, Math.-Naturwiss. R.; 25. Jg. (1976), H. 1.

[8] Jentsc日, L.: Die Greensche Matrix für zwei aneinander reibungsfrei gleitende elastische Halbräume mit versehiedenen Laméschen Moduln. ZAMM 58 (1978), 209-224.

[9] Jentsch, L.: Thermoelastostatik stückweise homogener Körper mit gleitenden Einschlüssen. Demonstratio Mathematica, Vol. XII, No. 1 (1979), $261-280$.

[10] Jentscir, L.: Die elastostatischen Greenschen Tensoren 1. und 4. Art für den Halbraum ils Grenzfälle éines Tensors für zwei aneinandergrenzende Halbräume mit verschiedenen Laméschen Moduln. Seminar of Institute of Applied Mathematics Reports 12-13 (1978), Tbilisi University.

[11] Jentsch, L., und J. MadL : Zur Elastizitäts- und Thermoelastizitätstheorie. Schriftenreihe Mathematische Forschung bei der Akademie der Wissensch. der DDR, Bd: 4. Akademie-Verlag: Berlin 1980.

[12] Jentsch, L.: ZZur Theorie der Wärmespannungen in Bimetallkörpern. Wiss. Z. KarlMarx-Univ: Leipzig, Math.-Naturw. R., 29. Jg. (1980), H. 1, 49-58.

[13] Jentscr, L. : Der Greensche Kontakttensor der Elastostatik für zwei fest verbundene Halbebenen. ZAMM 61, H. 7 (1981), 339-340.

[14] КатммадзЕ, Р. Г.: Новая контактная задаяа теориі упругости. Сообцения Акад. Наук Груз. ССР 95 (1979), 수 1, 49-52.

[15] Катммадз, Р. Г.: О существовании решения одной контактной задати теории упругости. Сообщения Акад. Наук Груз. ССР 96 (1979), 구 2, 305-307.

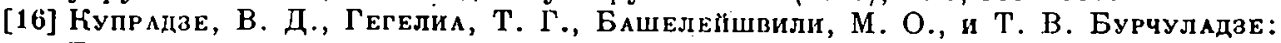
Трехмерные задачи математической теории упругости и термоупругости. Издательство Наука: Москва 1976.

[17] КупрАдзЕ, В. Д.: О контактных задачах теории упругости. Диффференциальные , уравнения, Т. XVI, 수 2, 1980, 293-310.

[18] MaUL, J.: Eine einheitliche Methode zur Lösung der ebenen Aufgaben der linearen Elastostatik. Schriftenreihe des ZIMM bei der Akademie der Wissensch. der DDR, Heft 24. Ákademie-Verlng: Berlin 1976. 
[19] MaUL, J.: Beiträge zur Existenztheorie gemischter Randwert- und Kontaktaufgaben der klassischen und mikropolaren Elàstizitätstheorie und Thermoelastizitätstheorie. Reprint Karl-Marx-Univ. Leipzig 1977.

[20] Mromrn, S. G., und S. Prössuorf: Singuläre Integraloperatoren. Ákademie-Verlag: Berlin 1980.

[21] Мусхелишвили, Н. И., Некӧторые основные задачи математической теории упругости. Москва $196 \dot{6}$.

$\because \quad$ Erschienen in deutschèr Úbersetzung:

Musschelischwilr, N. I.: Einige Grundaufgaben zur mathematischen Elastizitätstheorie. VEB Fachbuchverlag: Leipzig 1971.

[22]. Мусхелишвияли, Н. И.: Сингулярные иптегральные уравнения. Москва 1962.

[23] Petrowski, l. G.: Vorlesungen über partielle Differentialgleichungen. B. G. Teubner: Leipzig 1955.

[24] Prössdorf, S.: Einige Klassen singulärer Gleichungen. Akademie-Verlag: Berlin 1974.

[25] Theoćaris, P. S.: On the singular stress field at a multiwedge corner. Rev. Roumaine . Sci. Tech. Sér. Méc. Appl. 23 (1978), no. 5, 673-685.

Manuskripteingang:, 28.07. 1981

\section{VERFASSER:}

Prof. Dr. sc. nat. Lothar Jeniscir

Sektion Mathematik der 'Technischen Hochschule

- DDR -9010 Karl-Marx-Sta.dt, Reichenhainer Str. 39-41 